JOURNAL OF THE AMERICAN MATHEMATICAL SOCIETY

Volume 20, Number 2, April 2007, Pages 441-465

S 0894-0347(06)00537-6

Article electronically published on July 11, 2006

\title{
MINIMAL SURFACES WITH THE AREA GROWTH OF TWO PLANES: THE CASE OF INFINITE SYMMETRY
}

\author{
WILLIAM H. MEEKS III AND MICHAEL WOLF
}

\section{INTRODUCTION}

Consider a properly immersed minimal surface $M$ in $\mathbb{E}^{3}$ with area $A(r)$ in balls $B(r)$ of radius $r$ centered at the origin. By the monotonicity formula, the function $\bar{A}(r)=\frac{A(r)}{r^{2}}$ is monotonically increasing. We say that $M$ has area growth constant $A(M) \in(0, \infty]$, if $A(M)=\lim _{r \rightarrow \infty} \bar{A}(r)$. Note that under a rigid motion or homothety $M^{\prime}$ of $M$, the number $A(M)=A\left(M^{\prime}\right)$, and so, $A(M) \geq \pi$, which is the area growth constant of a plane. We say that $M$ has quadratic area growth, if $A(M)<\infty$.

Basic results in geometric measure theory imply that for any $M$ with quadratic area growth and for any sequence of positive numbers $t_{i} \rightarrow 0$, the sequence of homothetic scalings $M(i)=t_{i} M$ of $M$ contains a subsequence that converges on compact subsets of $\mathbb{E}^{3}$ to a limit minimal cone $C$ in $\mathbb{E}^{3}$ over a geodesic integral varifold in the unit sphere $\mathbb{S}^{2}$, which consists of a balanced finite configuration of geodesic arcs with positive integer multiplicities. $C$ is called a limit tangent cone at infinity to $M$.

In 1834, Scherk [15] discovered a singly-periodic embedded minimal surface $\mathcal{S}_{\frac{\pi}{2}}$ in $\mathbb{E}^{3}$ with quadratic area growth constant $2 \pi$. Away from the $x_{3}$-axis, Scherk's surface is asymptotic to the union of the $\left(x_{1}, x_{3}\right)$-plane and the $\left(x_{2}, x_{3}\right)$-plane. Geometrically, Scherk's singly-periodic surface may be viewed as the desingularization of these two orthogonal planes, which form its unique limit tangent cone at infinity. More generally, Scherk [15] (also see Karcher [2]) defined a one-parameter deformation $\mathcal{S}_{\theta}, \theta \in\left(0, \frac{\pi}{2}\right]$, of his original surface $\mathcal{S}_{\frac{\pi}{2}}$, which are also called Scherk surfaces and which may be viewed as the desingularization of two vertical planes with an angle $\theta$ between them. The limit tangent cone at infinity to $\mathcal{S}_{\theta}$ consists of the union of these planes. We remark that under appropriate homothetic scalings, the surfaces $\mathcal{S}_{\theta}$ converge to a catenoid as $\theta \rightarrow 0$. Note that a catenoid has a plane of multiplicity two as its limit tangent cone at infinity.

In [7, Meeks presented the following two conjectures related to minimal surfaces with quadratic area growth.

Received by the editors March 10, 2005.

2000 Mathematics Subject Classification. Primary 53A10; Secondary 32G15.

The first author was partially supported by NSF grant DMS-0405836.

The second author was partially supported by NSF grants DMS-9971563 and DMS-0139887.

Any opinions, findings, and conclusions or recommendations expressed in this publication are those of the authors and do not necessarily reflect the views of the NSF.

(C)2006 American Mathematical Society Reverts to public domain 28 years from publication 
Conjecture 1 (Unique Limit Tangent Cone Conjecture; see Conjecture 3.15 in [7]). A properly immersed minimal surface in $\mathbb{E}^{3}$ of quadratic area growth has a unique limit tangent cone at infinity.

Conjecture 2 (Scherk Uniqueness Conjecture; see Conjecture 3.14 in [7). A connected properly immersed minimal surface $M$ in $\mathbb{E}^{3}$ with quadratic area growth constant $A(M)<3 \pi$ must be a plane, a catenoid or a Scherk singly-periodic minimal surface $\mathcal{S}_{\theta}$, for some $\theta \in\left(0, \frac{\pi}{2}\right]$.

The main goal of this paper is to prove Conjecture 2 under the additional hypothesis of infinite symmetry.

Theorem 1. A connected properly immersed minimal surface in $\mathbb{E}^{3}$ with infinite symmetry group and area growth constant less than $3 \pi$ is a plane, a catenoid or a Scherk singly-periodic minimal surface.

We view Theorem 1 as a first step in resolving Conjecture 2. We hope that some of the ideas used in the proof of Theorem 1 will eventually lead to a proof of this more general conjecture and that such a proof will in turn lead to a positive solution of the following fundamental singularities question.

Conjecture 3 (Isolated Singularities Conjecture; see Conjecture 4 in [6]). Suppose $M$ is a minimal surface in a closed geodesic ball $B$ in a Riemannian three-manifold such that $\partial M \subset \partial B$ and $M$ is properly embedded in $B$ punctured at the center of the ball. Then $M$ extends across the puncture to a smooth compact embedded minimal surface in $B$.

Suppose now that $M$ is a properly immersed minimal surface in $\mathbb{E}^{3}$ with infinite symmetry group. Then $M$ is either a surface of revolution, and so, is a catenoid, or $M$ is invariant under a screw motion symmetry with possibly trivial rotational part and the symmetry acts in an orientation-preserving manner on $M$. In [12], Meeks and Rosenberg studied properly embedded minimal surfaces $M$ in $\mathbb{E}^{3}$, which are invariant under a group $\mathbb{Z}$ of isometries generated by a screw motion symmetry with vertical axis and whose quotient surface $\bar{M}$ in the flat three-manifold $\mathbb{E}^{3} / \mathbb{Z}$ has finite topology. They proved that the ends of such an $\bar{M}$ are asymptotic to horizontal planes, vertical flat half annuli (quotients of half-planes in $\mathbb{E}^{3}$ ) or helicoid ends in $\mathbb{E}^{3} / \mathbb{Z}$. Thus, if $M$ has quadratic area growth and $\bar{M}$ has finite topology (finitely generated fundamental group), then $\bar{M}$ must have ends asymptotic to vertical half annuli. Such annular ends of $\bar{M}$ are called Scherk ends, because the singly-periodic quotients $\overline{\mathcal{S}}_{\theta}$ of the classical Scherk examples, $\mathcal{S}_{\theta} \subset \mathbb{E}^{3}, \theta \in\left(0, \frac{\pi}{2}\right]$, have this type of end. It follows that $M$ is also invariant under a pure translation, and so, after a rigid motion and homothety, we will assume that $\mathbb{Z}=\{(0,0, n) \mid n \in \mathbb{Z}\}$ acts by translation on $\mathbb{E}^{3}$ in this case.

The next theorem is the key result that we need to prove Theorem 1 .

Theorem 2. The Scherk minimal surfaces $\overline{\mathcal{S}}_{\theta}$ are the unique connected minimal surfaces in $\mathbb{E}^{3} / \mathbb{Z}$ with four Scherk ends.

To our knowledge, this theorem is the first uniqueness result for singly-periodic Scherk surfaces of genus greater than 1 . The case of genus 0 was shown by Meeks and Rosenberg [10, and the case of genus 1 was carried out in the doctoral thesis of Hai-Ping Luo [5]. 
We emphasize a corollary of Theorem 2 by noting that, if $\bar{M} \subset \mathbb{E}^{3} / \mathbb{Z}$ has ends asymptotic to vertical half annuli, then its lift to $\mathbb{E}^{3}$, in the complement of a vertical cylinder, is asymptotic to four half-planes. Thus, we may regard such a surface as a periodic minimal desingularization of the intersection of two planes and rephrase Theorem 2 as:

Corollary 1. The Scherk minimal surfaces $\mathcal{S}_{\theta}$ are the unique connected periodic minimal desingularizations of the intersections of two planes.

Our proof of Theorem 1 is broken up into a series of propositions, which appear in sections of the manuscript. Let $\bar{M} \subset \mathbb{E}^{3} / \mathbb{Z}$ be a connected properly immersed minimal surface with four Scherk ends. The lifted surface $M \subset \mathbb{E}^{3}$ then has quadratic area growth constant $2 \pi$. Applying the monotonicity formula for area to $M$ at a possible point of self-intersection, we see that $\bar{M}$ is an embedded minimal surface. In section 2 , we prove that the corresponding $M$ has two vertical planes of Alexandrov symmetry, just as the classical Scherk examples have. These planes decompose $M$ into four nonempty closed simply connected regions; we study two holomorphic one-forms underlying the Weierstraß representation of $M$. These forms naturally define flat structures on each of these four regions in $M$, and these flat structures develop to one of the two complements of a zigzag in $\mathbb{E}^{2}$. In section 3 , we prove a local rigidity theorem for the surfaces $M$ with fixed angle $\theta$ between their Scherk ends. The implicit function theorem then asserts that, in terms of the angle map between the Scherk ends $\theta: \mathcal{M}_{\varkappa} \rightarrow\left(0, \frac{\pi}{2}\right]$, defined on the moduli space $\mathcal{M}_{\varkappa}$ of examples $\bar{M}$ of genus $\varkappa$ in $\mathbb{E}^{3} / \mathbb{Z}$ (defined up to congruence), we have that every component of $\mathcal{M}_{\varkappa}$ is a nontrivial curve $C$ and $\left.\theta\right|_{C}: C \rightarrow \theta(C) \subset\left(0, \frac{\pi}{2}\right]$ is a diffeomorphism. In section 4 , we prove that $\theta: \mathcal{M}_{\varkappa} \rightarrow\left(0, \frac{\pi}{2}\right]$ is proper, and so, the map $\left.\theta\right|_{C}: C \rightarrow\left(0, \frac{\pi}{2}\right]$ is a diffeomorphism. In section 5 , we prove that for $\alpha$ close to 0 , the preimage $\theta^{-1}(\alpha)$ is one of the Scherk examples $\overline{\mathcal{S}}_{\alpha}$. Thus, $\mathcal{M}_{\varkappa}$ contains only one component, which is the component of Scherk examples. This result proves Theorem 2

In section 6 , we prove that if $M$ is a properly embedded minimal surface in $\mathbb{E}^{3}$ with $A(M)<3 \pi$, then either $A(M)=\pi$ and so $M$ is a plane or $A(M)=2 \pi$. Under the assumption that $M$ has infinite symmetry group, we then prove that $M$ is either a plane, a catenoid or $M$ is invariant under a group $\mathbb{Z}$ of translations with $\bar{M}=M / \mathbb{Z}$ having finite topology. Then, our results from section 5 complete the proof of Theorem 1 .

Our basic strategy of proving Theorem 2 is to show that the angle map $\theta$ on the moduli space $\mathcal{M}_{\varkappa}$ is open and proper and that examples with small angle in $\mathcal{M}_{\varkappa}$ are Scherk; this strategy is motivated by the proofs of two previous uniqueness theorems in the literature. Lazard-Holly and Meeks [4] used this approach in their characterization of the family of Scherk doubly-periodic minimal surfaces $\widetilde{\mathcal{S}}_{\theta}, \theta \in\left(0, \frac{\pi}{2}\right]$, which are also parametrized by the angle between the ends of their quotient surfaces, as being the only properly embedded minimal surfaces in $\mathbb{E}^{3}$ with genus 0 quotients. A similar approach was also used by Meeks, Pérez and Ros 9 to characterize the one-parameter family of Riemann minimal examples, the helicoid and the plane, as being the only properly embedded periodic genus 0 minimal surfaces in $\mathbb{E}^{3}$. In another direction, Pérez and Traizet [14] have recently classified the properly embedded singly-periodic minimal surfaces with quotient surfaces having 
genus 0 and finite topology; their classification theorem has similar structural attributes and they prove that these surfaces are precisely the Scherk towers defined earlier by Karcher [2. Their classification result then leads to the classification [13] of properly embedded doubly-periodic minimal surfaces in $\mathbb{E}^{3}$ whose quotients have genus 1 and parallel annular ends in $\mathbb{T} \times \mathbb{R}$, where $\mathbb{T}$ is a flat torus. We remark that this last classification result implies that these genus 1 minimal surfaces are the same examples which were defined by Karcher in [3].

Finally, we remark that the idea used in our proof of Theorem 2 of demonstrating the local rigidity of a minimal surface in a moduli space of flat structures is a cornerstone of the work of Weber and Wolf $([19,20,21])$ in their Teichmüllertheoretic approach to existence problems in minimal surface theory.

\section{Existence of Alexandrov planes of REFlECTIONAL SyMMETRY}

The following proposition is well known to experts in the field and the proof (unpublished) we give is due to Harold Rosenberg.

Proposition 3. Let $M \subset \mathbb{E}^{3}$ be a properly embedded minimal surface invariant under translation by the vectors $\mathbb{Z}=\{(0,0, n) \mid n \in \mathbb{Z}\}$ and such that the quotient surface $\bar{M}=M / \mathbb{Z}$ has four Scherk ends and the genus of $\bar{M}$ is $\varkappa$. Let $A_{x_{1}}$ and $A_{x_{2}}$ be the vertical annuli parallel to the $x_{1}$ - and $x_{2}$-axes, respectively, which are quotients of the vertical $\left(x_{1}, x_{3}\right)$ - and the $\left(x_{2}, x_{3}\right)$-planes, respectively by the $\mathbb{Z}$ action.

Then:

(1) After rigid motion, we may assume $\bar{M}$ is invariant under reflection in the vertical annuli $A_{x_{1}}, A_{x_{2}}$, which each intersect $\bar{M}$ orthogonally in $\varkappa+1$ strictly convex simple closed curves contained in the respective annuli.

(2) The regions of $\bar{M}$ on either side of $A_{x_{1}}$ or $A_{x_{2}}$ are graphs over their projections to the respective annuli. In particular, the $\left(x_{1}, x_{3}\right)$-plane and the $\left(x_{2}, x_{3}\right)$-plane are Alexandrov planes of reflexive symmetry for $M$, after a rigid motion of $M$.

The situation is illustrated in Figure 1.

Proof. Consider the flux vectors

$$
v_{i}=\int_{\gamma_{i}}\left(\nabla x_{1}, \nabla x_{2}\right), i=1,2,3,4
$$

defined for oriented loops $\gamma_{1}, \gamma_{2}, \gamma_{3}, \gamma_{4}$ around the four cyclically ordered Scherk ends of $\bar{M}$. Since each of these vectors is a unit vector and the sum of these vectors is zero by the divergence theorem, we see that after a rotation of $M$ around the $x_{3}$-axis, we must have $v_{1}=(\cos \alpha, \sin \alpha), v_{2}=(\cos \alpha,-\sin \alpha), v_{3}=-v_{1}$ and $v_{4}=-v_{2}$, where $\alpha \in\left(0, \frac{\pi}{4}\right]$. Note that the Scherk ends of $\bar{M}$ are asymptotic to ends $A_{1}, A_{2}, A_{3}, A_{4}$, of flat vertical annuli, where $v_{i}$ is parallel to $A_{i}$.

Now consider the family $E(1, t)$ of vertical annuli, which are parallel to $A_{x_{1}}$ and pass through the point $(0, t, 0)$, for $t \in \mathbb{R}$. Let $R(1, t): \mathbb{R}^{2} \times \mathbb{R} / \mathbb{Z} \rightarrow \mathbb{R}^{2} \times \mathbb{R} / \mathbb{Z}$ denote reflection across $E(1, t)$, let $\bar{M}_{+}(1, t)$ denote the portion of $\bar{M}$ on the side of $E(1, t)$ which contains large positive $x_{1}$ valued points of $\bar{M}$ and let $\bar{M}_{-}(1, t)=$ $R(1, t)\left(\bar{M}_{+}(1, t)\right)$. Note that for $t>0$ sufficiently large, the surface $\bar{M}_{+}(1, t)$ consists of two almost flat annular end representatives for $\bar{M}$ and $\partial \bar{M}_{+}(1, t)=$ $\partial \bar{M}_{-}(1, t)=\bar{M} \cap \bar{M}_{-}(1, t)$. 


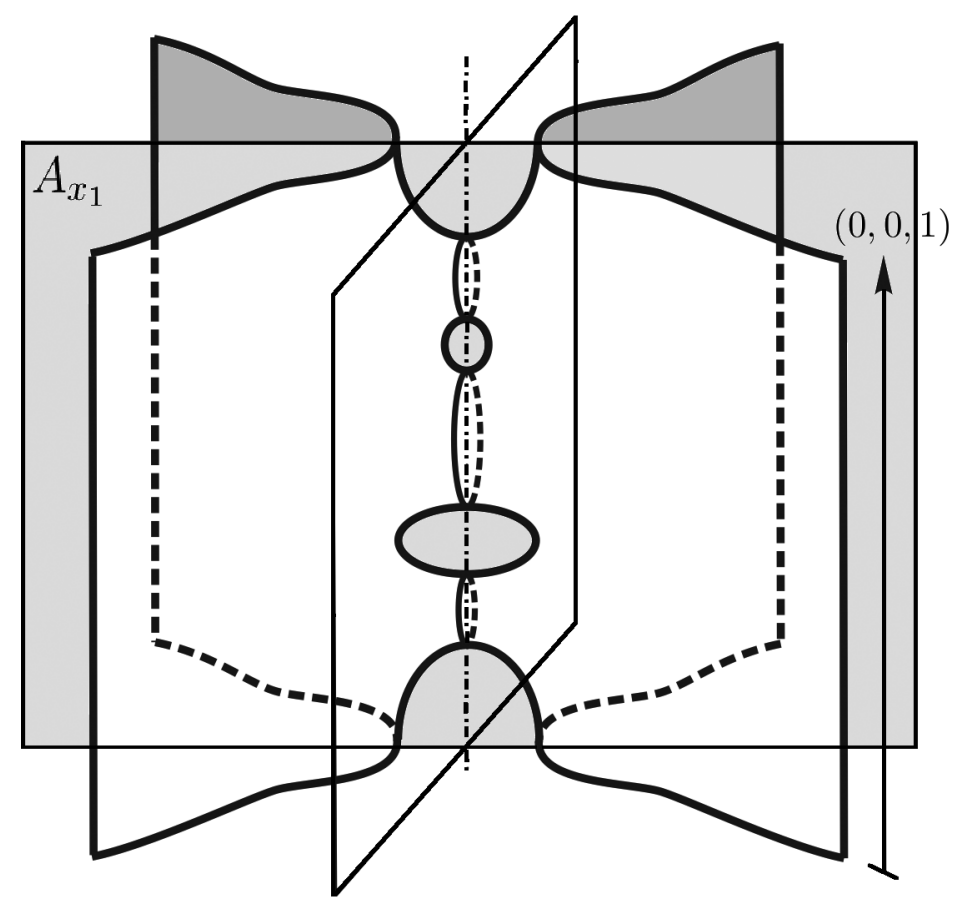

Figure 1. The vertical annuli $A_{x_{1}}$ and $A_{x_{2}}$ meet $\bar{M}$.

Define $t_{1}$ to be the infimum of the values $t$ such that $\bar{M}_{+}(1, t)$ is a nonnegative graph with bounded gradient over its projection to $E(1, t)$ and $\partial \bar{M}_{-}(1, t)=\bar{M} \cap$ $\bar{M}_{-}(1, t)$. (The set of such $t$ is nonempty because the components of $\bar{M}_{+}(1, t)$ are rapidly asymptotic to flat annuli.) By the interior maximum principle and the Hopf maximum principle applied along $\partial \bar{M}_{-}\left(1, t_{1}\right)$, we observe that $\bar{M}_{+}\left(1, t_{1}\right)$ is a graph over its projection to $E\left(1, t_{1}\right), R\left(1, t_{1}\right)(\bar{M})=\bar{M}$ and that $\bar{M}$ is orthogonal to $E\left(1, t_{1}\right)$. This observation is just the standard one that arises in the application of the Alexandrov reflection argument when one takes into account the maximum principle at infinity [11, which guarantees that an end of $\bar{M}_{-}\left(1, t_{1}\right)$ cannot be asymptotic to an end of $\bar{M}$ unless $\bar{M}_{-}\left(1, t_{1}\right) \subset \bar{M}$.

Note that $\partial \bar{M}_{+}\left(1, t_{1}\right)$ consists of a finite number of simple closed curves in $E\left(1, t_{1}\right)$ since it has compact boundary. Furthermore, $\bar{M}_{+}\left(1, t_{1}\right)$ is a planar domain with two ends since it is a graph over a proper noncompact planar domain in the annulus $E\left(1, t_{1}\right)$ with two ends. Since the Euler characteristic $\chi(\bar{M})=$ $2(1-\varkappa)-4=-2-2 \varkappa$, then $\partial \bar{M}_{+}\left(1, t_{1}\right)=\bar{M} \cap E\left(1, t_{1}\right)$ consists of $\varkappa+1$ simple closed curves. Since these curves are planar lines of curvature on $\bar{M}$ and $\bar{M}_{+}\left(1, t_{1}\right)$ is a graph over its projection to $E\left(1, t_{1}\right)$, then the simple closed curves in $\partial M_{+}(1, t)$ are strictly convex curves bounding disks in $E\left(1, t_{1}\right)$.

Similarly, we have for some $t_{2}$ a related annulus $E\left(2, t_{2}\right)$ parallel to $A_{x_{2}}$, which is an Alexandrov annulus of symmetry for $\bar{M}$. After a fixed translation of $\bar{M}$, we may assume that the circle $E\left(1, t_{1}\right) \cap E\left(2, t_{2}\right)$ is $(0,0) \times \mathbb{R} / \mathbb{Z} \subset \mathbb{R}^{2} \times \mathbb{R} / \mathbb{Z}$. The proposition now follows. 


\section{The Angle map $\theta: \mathcal{M}_{\varkappa} \rightarrow\left(0, \frac{\pi}{2}\right]$ IS A LOCAL DIfFEOMORPhism}

Let $\mathcal{M}_{\varkappa}$ denote the moduli space of examples $\bar{M}$ of genus $\varkappa$ in $\mathbb{R}^{3} / \mathbb{Z}$ (defined up to congruence). For $\bar{M} \in \mathcal{M}_{\varkappa}$, let $\theta(\bar{M})=\theta(M)$ denote the angle between the Scherk ends of $M$.

Our goal in this section is to prove the following result.

Proposition 4. For any component $C$ in $\mathcal{M}_{\varkappa}$, the image $\theta(C)$ is an open subset of $\left(0, \frac{\pi}{2}\right]$ and $\left.\theta\right|_{C}: C \rightarrow \theta(C)$ is a diffeomorphism.

The proof of Proposition 4 will depend on the following lemma.

Lemma 5. If $\bar{M} \in \mathcal{M}_{\varkappa}$ with $\theta(\bar{M})=\theta_{0}$, then $\bar{M}$ is locally rigid, i.e., there are no deformations of $\bar{M}$ though a family of minimal surfaces in $\mathcal{M}_{\varkappa}$ with the same angle $\theta_{0}$.

The proof of the lemma occupies the rest of this section, and so has the appearance of being quite a lengthy argument; however, most of the passage is devoted to describing the perspective that we will take when we undertake the relatively short computation towards the end of subsection 3.3 .2

Our plan is the following. In the remainder of this introductory portion, we recall the Weierstraß representation in the notation we will be using throughout. Then in the next subsection, we study the resulting forms in terms of the Euclidean developments of their associated flat metrics: we will carefully describe these "flat structures" at that time. In subsection 3.2 , we will describe the quantity $\theta(M)$ as a geometric invariant of those flat structures. The final piece of background information is developed in subsection 3.3: there we discuss how the flat structures deform, and we relate the deformations of the flat structures to conformal deformations of the underlying Riemann surfaces. With all of this background then behind us, the proof of the lemma is relatively quick: in subsection 3.3.2, we compute that any deformation of a Weierstraß pair of forms that preserves the period conditions must then destroy the agreement of the underlying conformal structures. That completes the proof of the lemma, with Proposition 4 then following easily.

Before we begin the proofs, we need to recall the Weierstraß representation and set some notation. Recall that for a Riemann surface $\mathcal{R}$ with a holomorphic function $g$ and a holomorphic form $d h$ (not necessarily exact, despite the notation), we may define (locally defined because of possible periods) a conformal branched minimal immersion via a map $F: \mathcal{R} \rightarrow \mathbb{E}^{3}$ by

$$
z \mapsto \operatorname{Re} \int_{\rho_{0}}^{z}\left(\frac{1}{2}\left(g-\frac{1}{g}\right) d h, \frac{i}{2}\left(g+\frac{1}{g}\right) d h, d h\right) .
$$

For this surface, the function $g$ will be the Gauss map (postcomposed with stereographic projection) and $d h$ will be the complexified differential of the third coordinate in $\mathbb{E}^{3}$. Conversely, given a conformal minimal immersion $F: \mathcal{R} \rightarrow \mathbb{E}^{3}$ with Gauss map $g$ and complexified differential $d h$ of the third coordinate, the surface may be represented by the expression (11). The induced metric on the minimal surface is given by

$$
d s_{F(\mathcal{R})}=\frac{1}{2}\left(|g|+\frac{1}{|g|}\right)|d h| ;
$$

thus a regular minimal surface will have zeroes of $d h$ of order $n$ balanced by poles or zeroes of $g$ of the same order. 
The global problem for producing minimal surfaces is that of well-definedness: analytic continuation around a cycle must leave the map unchanged. Thus we require

$$
\begin{aligned}
& \operatorname{Re} \int_{\gamma} \frac{1}{2}\left(g-\frac{1}{g}\right) d h=\operatorname{Re} \int_{\gamma} \frac{i}{2}\left(g+\frac{1}{g}\right) d h \\
& =\operatorname{Re} \int_{\gamma} d h=0
\end{aligned}
$$

for every cycle $\gamma \subset \mathcal{R}$. Note that the vanishing of real periods in the first of the above equations is equivalent to

$$
\int_{\gamma} g d h=\overline{\int_{\gamma} \frac{1}{g} d h}
$$

for every cycle $\gamma \subset \mathcal{R}$.

With this background in hand, we may begin the proof of Lemma 5 .

Proof. Let $M$ be the lift of $\bar{M}$ to $\mathbb{E}^{3}$. Let $g$ and $d h$ denote the Weierstraß data of $M$ in the standard notation. These combine to define the forms $g d h$ and $\frac{1}{g} d h$, which we restrict to one of the fundamental domains of the surface with respect to the $\mathbf{Z}_{2} \oplus \mathbf{Z}_{2}$ group of Alexandrov reflections guaranteed to exist by Proposition 3 .

3.1. The shape of the developments of $|g d h|$ and $\left|\frac{1}{g} d h\right|$. The fundamental domain described above is planar, and as the group elements act as isometries of the singular flat metrics $|g d h|$ and $\left|\frac{1}{g} d h\right|$, the forms $g d h$ and $\frac{1}{g} d h$ develop (injectively, as there are no finite or infinite branch points) to planar Euclidean domains, say $\Omega_{g d h}$ and $\Omega_{g^{-1} d h}$ bounded by a "zigzag" boundary, as we describe in just a moment. Before we do that, however, we note that we already see that as the fundamental domain is periodic with respect to a translation (in $\mathbb{E}^{3}$ ), which is an isometry of $|g d h|$ and $\left|\frac{1}{g} d h\right|$ as well, then the developed image of the domain is invariant by a cyclic group of translational isometries (of the plane).

The situation is illustrated in Figure 2 .

Much of our attention in this section will be focused on the boundaries of the developed domains $\Omega_{g d h}$ and $\Omega_{g^{-1} d h}$, as those boundaries contain much of the geometry of the surface $M$. Indeed, from the geometry of $M$, we recognize the basic shape of the zigzag boundary of the developed image in $\mathbb{E}^{2}$. To draw this boundary, begin by drawing an arc downwards for some distance at a slope of -1 . Then make a left turn and draw a segment upwards at a slope of +1 . Then draw downwards along a segment of slope -1 , etc. Continue drawing in this way $n=2 \varkappa+2$ segments ( $n$ is even and $\varkappa$ is the genus of $M$ ) and then repeat the pattern indefinitely, both backwards and forwards. This construction is meant to determine the $g d h$ structure (the portion of the plane above the zigzag) for the surface $M$.

The $\frac{1}{g} d h$ structure is then determined by the requirement that its periods should be conjugate to those of the $g d h$ structure, i.e., $\int_{\gamma} g d h=\overline{\int_{\gamma} \frac{1}{g} d h}$ for all cycles $\gamma \subset M$. So we do this: on another plane, we draw another zigzag, so that the portion of the plane above that zigzag will correspond to the $\frac{1}{g} d h$ structure. The 


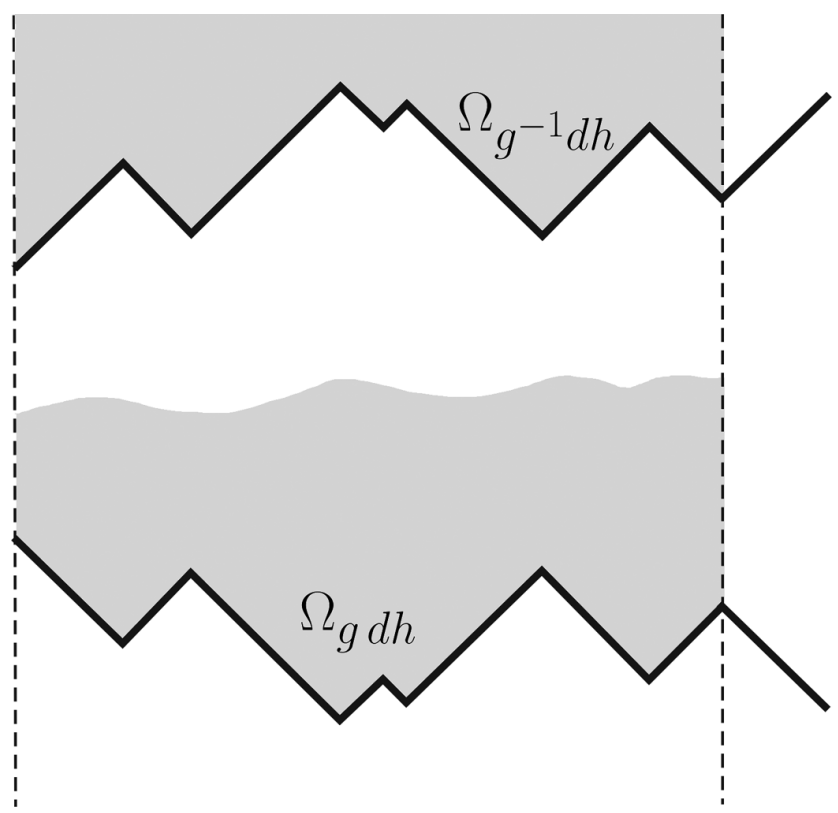

Figure 2. The two domains, $\Omega_{g d h}$ and $\Omega_{g^{-1} d h}$, drawn above their boundaries

conjugate period requirement is that we draw it as follows: we draw the first arc at a slope of +1 and of exactly the same length as the first segment on the first $(g d h)$ zigzag. Then we draw the second arc at a slope of -1 of exactly the same length as the second segment on the first zigzag. We draw the third arc at slope +1 and of the same length as the third segment of the first zigzag. We continue this construction for all $n$ segments and then repeat in both directions to guarantee symmetry by an infinite group of translations (i.e., isometries of the range $\mathbb{E}^{2}$ of development).

We assert that the developments $\Omega_{g d h}$ and $\Omega_{g^{-1} d h}$ of a fundamental domain for the action of the $\mathbf{Z}_{2} \oplus \mathbf{Z}_{2}$ group of Alexandrov reflections on $M$ have the forms described above. In effect, we have to prove two statements to justify this: first we need to show that the boundaries of those domains $\Omega_{g d h}$ and $\Omega_{g^{-1}} d h$ are piecewise straight, and then we need to show that those straight edges meet at angles that alternate between $\frac{\pi}{2}$ and $\frac{3}{2} \pi$. For the first claim, note that those Alexandrov reflections are isometries of the flat singular metrics $|g d h|$ and $\left|\frac{1}{g} d h\right|$, and that the boundary of the fundamental domain is fixed by the isometry. But as a smooth fixed set of an isometry is totally geodesic, and the metrics $|g d h|$ and $\left|\frac{1}{g} d h\right|$ are flat and smooth away from the poles and zeroes of $g$, we see that the smooth components of the boundary of the developed images $\left(\Omega_{g d h}\right.$ and $\left.\Omega_{g^{-1} d h}\right)$ of the fundamental domain of $M$ are bounded by straight lines. Next observe that at the endpoints of those geodesic segments (those endpoints corresponding to the $2 \varkappa+2$ points where 
$\bar{M}$ meets the intersection $A_{x_{2}} \cap A_{x_{1}}$ described in Proposition 3), the forms $g d h$ and $\frac{1}{g} d h$ alternate between being regular and having second-order zeroes. (Also, one of those two forms has a double zero at such a point if and only if the other one of the forms is regular there.) Of course, the developed image of a holomorphic one-form with a zero of order $k$ has a cone point with cone angle $2 \pi(k+1)$; as these endpoints of the straight lines are fixed points of all four of the reflections, we see that one-quarter of the cone-angles will be visible in one of the fundamental domains. Thus the boundaries will be composed of straight lines, meeting at angles that alternate between $\frac{\pi}{2}$ and $\frac{3}{2} \pi$, with angles at corresponding points of $\Omega_{g d h}$ and $\Omega_{g^{-1} d h}$ disagreeing, as claimed.

Because the $g d h$ and $\frac{1}{g} d h$ structures are defined on the same Riemann surface, there is a conformal map between those planar domains (above the corresponding zigzag).

In all of this, we have ignored the flat structure for the form $d h$. This is because, following the same procedure for the development of $d h$ as we did for the developments of $\Omega_{g d h}$ and $\Omega_{g^{-1} d h}$, we see that the form $d h$ develops on a fundamental domain to a domain with piecewise straight edges meeting at angles of $\pi$. Thus the developed image is a (geometric) half-plane with a periodic collection of distinguished points on the boundary. As any such domain satisfies the (vertical) period condition that the distinguished points lie horizontally parallel to each other, we see that any such domain will satisfy the relevant period condition, and there is no restriction on the geometry of this domain. This geometric fact corresponds to the observation that the developed domain doubles to a geometric sphere, so that the form $d h$ is exact. From both points of view, we cannot expect to glean much information from the period condition for $M$ on $d h$.

3.2. The angle between the ends. Having described the geometric structure of the developments $\Omega_{g d h}$ and $\Omega_{g^{-1} d h}$ of the defining Weierstraß forms $g d h$ and $\frac{1}{g} d h$ of $M$, our next goal is to describe the moduli space $\mathcal{M}\left(\theta_{0}\right)$ of candidates for surfaces with asymptotic angle fixed at $\theta=\theta_{0}$. To do this, we need to recognize the angle between those asymptotic planes in the zigzags, as our moduli space will be defined in terms of zigzags.

The surface $M$ in $\mathbb{R}^{3}$ is periodic, invariant by a group of translations generated by a vertical translation of distance, say, $\ell_{M}(e)$.

Proposition 6. There is a function $m(\theta)$ which is strictly monotone in $\theta$ so that if the Scherk ends of $M$ make an angle of $\theta$ with one another, then the zigzag boundaries of the domains $\Omega_{g d h}$ and $\Omega_{g^{-1}}$ dh are invariant by a group of translations generated by $\left\langle z \mapsto z+\ell_{M}(e) \exp (i m(\theta))\right\rangle$, where $\ell_{M}(e)$ is the length of the translation vector of $M$ and $i=\sqrt{-1}$.

We begin by considering the quotient of the surface by a single vertical plane of symmetry. A single fundamental domain of the quotient has flux $\vec{F}$ across this boundary equal to the flux across its ends, the latter given by

$$
\vec{F}=(2 \cos (\theta / 2)) h \vec{n} \text {. }
$$

Here $\theta$ is the angle between the ends, $h=\ell_{M}(e)$ is the (normalized) height of the fundamental domain, and $\vec{n}$ is the (appropriate) normal to the reflective planes. Of course, the pair of ends of the domain is homologous to the $\varkappa+1$ closed curves 
$\left\{\gamma_{1}, \ldots, \gamma_{\varkappa+1}\right\}$ of intersection between the plane and the surface. As the surface is orthogonal to the plane along these curves, the flux across those curves is given by

$$
\begin{aligned}
\vec{F} & =\sum_{i=1}^{\varkappa+1} \ell_{M}\left(\gamma_{i}\right) \vec{n} \\
& =\left(\sum_{i=1}^{\varkappa+1} \int_{\gamma_{i}} \frac{1}{2}\left(|g|+\frac{1}{|g|}\right)|d h|\right) \vec{n} \\
& =\frac{1}{2}\left(\sum_{i=1}^{\varkappa+1}\left[\int_{\gamma_{i}}|g d h|+\int_{\gamma_{i}}\left|\frac{1}{g} d h\right|\right]\right) \vec{n}
\end{aligned}
$$

as the line element on the surface is given by $d s_{M}=\frac{1}{2}\left(|g|+\frac{1}{|g|}\right)|d h|$. Thus, we can rewrite the length of the flux vector as

$$
F=|\vec{F}|=\frac{1}{2} \sum_{i=1}^{\varkappa+1}\left[\ell_{|g d h|}\left(\gamma_{i}\right)+\ell_{\left|\frac{1}{g} d h\right|}\left(\gamma_{i}\right)\right]
$$

We now consider the quotient by action of reflection in the other vertical plane. As reflection about this plane is an isometry for the metrics $|g d h|$ and $\left|\frac{1}{g} d h\right|$, we see that we may rewrite the previous equation as

$$
F=\sum_{i=1}^{\varkappa+1}\left[\ell_{\Omega_{g d h}}\left(\Gamma_{i}\right)+\ell_{\Omega_{g^{-1} d h}}\left(\Gamma_{i}\right)\right] .
$$

Here, we multiply by two because we are only measuring lengths on a single fundamental domain of the $\mathbf{Z}_{2} \oplus \mathbf{Z}_{2}$ action (instead of in the pair of fundamental domains in the previous line), and we interpret $\gamma_{i}$ as having trace $\Gamma_{i}$ in that domain. Yet, by construction, the arcs $\Gamma_{i}$ are the arcs of the zigzag of $\Omega_{g} d h$ which all have the same slope, say +1 , or of $\Omega_{g^{-1}} d h$ with slope -1 . Since, by construction as well, we have

$$
\ell_{\Omega_{g d h}}\left(\Gamma_{i}\right)=\ell_{\Omega_{g}-1 d h}\left(\Gamma_{i}\right)
$$

we conclude

$$
F=2 \sum_{i=1}^{\varkappa+1} \ell_{\Omega_{g d h}}\left(\Gamma_{i}\right)
$$

We consider next the total translational displacement of the zigzag, i.e., the Euclidean distance between a point and its image under a generator of the translational isometry group of $\Omega_{g d h}$ or $\Omega_{g^{-1}} d h$. Elementary Euclidean geometry describes this quantity in terms of the segments of the zigzag, as follows. Note that if we project a zigzag along one of its directions, we obtain a segment composed of isometric images of the arcs of one slope, and if we project in the orthogonal direction, we obtain a segment composed of isometric images of the arcs of the other slope: the transla- 
tional displacement is the length of the hypotenuse of the right triangle with these two segments as legs. Now the total translational displacement is normalized by the requirement that the translational distance $\ell_{M}(e)$ of the end is fixed. If $\Gamma$ is an arc around an end of a fundamental domain (i.e., $\gamma$ is part of a circular loop around a puncture $e$ on $\bar{M})$, then $\ell_{M}(\Gamma)=\frac{1}{2}\left(\ell_{|g d h|}(\Gamma)+\ell_{\left|g^{-1} d h\right|}(\Gamma)\right)$. As $\Gamma$ tends towards the end $e$, we have that $\ell_{M}(\Gamma)$ tends to $\ell_{M}(e)$. Moreover, since the Gauss map $g$ converges to a unitary complex number, we have $\ell_{|g d h|}(\Gamma)=\ell_{\left|g^{-1} d h\right|}(\Gamma)+o(1)$. Thus, we see that $\ell_{M}(e)=\ell_{\Omega_{g d h}}(\Gamma)+o(1)$ (as $\Gamma$ tends towards the end $e$ ) and this fixes the translational displacement length.

In summary, from equations (4) and (5), we know that the total length, say $L_{+}(\theta)$, in $\Omega_{g d h}$ of the segments $\left\{\Gamma_{1}, \ldots, \Gamma_{\varkappa+1}\right\}$ (of slope +1 ) is a monotone function of $\theta \in(0, \pi)$, while the length of the total displacement vector of the segments in a fundamental domain has length fixed independently of the angle $\theta$. Now the difference between the endpoints of a fundamental domain of the zigzag is described as having length $\ell_{M}(e)$ and $\operatorname{argument}$ given $\operatorname{as} \arctan \left(\frac{L_{+}}{L_{-}}\right)-\frac{\pi}{4}$, where $L_{-}$is the total length of all of the segments of the zigzag of slope -1 . Thus, as $L_{-}^{2}+L_{+}^{2}=\ell_{M}(e)^{2}$, we see that the slope of the orbit of a point in $\Omega_{g d h}$ under the action of the cyclic group of translations is monotone in the asymptotic angle $\theta$ between the ends.

For the rest of the proof of Lemma 5 , we will assume that the angle between the ends is fixed. The passage above shows that this forces the zigzag boundary to have an orbit whose slope is $m\left(\theta_{0}\right)$, which is a well-defined constant depending only on $\theta_{0}$.

3.3. Deformations of orthodisks. In general, a domain of the topological type of the disk which is bounded by a zigzag with orthogonal edges is known as an orthodisk. An orthodisk has geometry described by the positions of its vertices $\left\{P_{i}\right\}$. Two orthodisks with conjugate edge vectors $\overrightarrow{P_{j} P_{j+1}}$ are called conjugate orthodisks. We have shown in section 3.1 how a minimal surface of the type we are considering in this paper gives rise to a conjugate pair of orthodisks. Moreover, such a conjugate pair of orthodisks is quite special, as the identity map on the minimal surface descends to a conformal map between the orthodisks which takes vertices on one orthodisk, say $\Omega_{g d h}$, to corresponding vertices on the other orthodisk, say $\Omega_{g^{-1} d h}$. In this subsection, we will study a moduli space of pairs of conjugate orthodisks; these pairs will usually not be related by a conformal map which preserves corresponding vertices.

To introduce this space, consider a surface $\bar{M} \in \mathcal{M}_{\varkappa}$ with $\theta(\bar{M})=\theta_{0}$, as described in the statement of Proposition [6] Then the domains $\Omega_{g d h}$ and $\Omega_{g^{-1} d h}$ for $\bar{M}$ have zigzag boundaries with slopes $m\left(\theta_{0}\right)$ as described in the previous subsection. There is also then a $2 \varkappa$-dimensional family $\left\{\left(\Omega_{g d h}, \Omega_{g^{-1} d h}\right)\right\}$ of pairs of domains bounded by deformations of those zigzags invariant under the same group of Euclidean planar isometries as for $\bar{M}$ : of course, most of the pairs in this space will not be related by a conformal map which preserves corresponding vertices, as would be the case for the pair, say $Z_{0}$, of domains for $\bar{M}$.

We then consider a family $\left\{M_{t}\right\}$ of minimal surfaces containing $M=M_{0}$; these then induce, as above, a family $\left\{Z_{t}\right\}$ of such pairs of zigzags passing through $Z_{0}$, which would deform through domains with zigzag boundaries. Infinitesimally, then, we can compute the general form of the (infinitesimal) Beltrami differential for the $g d h$ domain and for its counterpart on the $\frac{1}{g} d h$ domain. This pair represents a 
tangent direction to the pair $\left(\Omega_{g d h}, \Omega_{g^{-1} d h}\right)$, construed to be a point in the product of Teichmüller spaces of the quotient domains.

With all of this background, the crux of the argument is to compute those Beltrami differentials. Before plunging into this computation, we outline the plan in the next three paragraphs. In particular, let us denote by, say $\nu$, the Beltrami differential prescribing the deformation on the $\frac{1}{g} d h$ structure $\Omega_{g^{-1}} d h$, and by, say $\mu$, the Beltrami differential parametrizing the deformation of the $g d h$ structure $\Omega_{g d h}$. We then pull back $\nu$ to the $g d h$ structure via the assumed conformal map $F$ and obtain a Beltrami differential $F^{*} \nu$. Then, if $\mu$ is the Beltrami differential for the $g d h$ structure, we compute (!) $F^{*} \nu=-\mu$.

The upshot of this result is that the two domains $\Omega_{g d h}$ and $\Omega_{g^{-1} d h}$ cannot remain conformally related for $t \neq 0$, unless the lengths of all of the segments are preserved, i.e., $g d h$ and $\frac{1}{g} d h$ do not change, which means that the family $\left\{M_{t}\right\}$ of minimal surfaces is infinitesimally unmoving.

In particular, from $F^{*} \nu=-\mu$, and since $-\mu$ is not equivalent to $\mu$ unless both are equivalent to zero, we conclude that neither structure has deformed. But one can check that this can only mean that no periods have changed, and so, the minimal surfaces $\left\{M_{t}\right\}$ have only deformed by a congruence/homothety. That concludes the argument, at least in outline form.

We need to formalize the previous discussion. Let $\Omega_{g d h}$ and $\Omega_{g^{-1} d h}$ denote the orthodisk structures for the forms $g d h$ and $\frac{1}{g} d h$, respectively. We are concerned with relating the Euclidean geometry of the orthodisks (which corresponds directly with the periods of the Weierstraß data, as in the construction above) to the conformal data of the domains $\Omega_{g d h}$ and $\Omega_{g^{-1} d h}$. From the discussion above, since a family of minimal surfaces $\left\{M_{t}\right\}$ will always give rise to a corresponding family $\left\{\left(\Omega_{g d h}(t), \Omega_{g^{-1} d h}(t)\right)\right\}$ of orthodisks, it is clear that the allowable infinitesimal motions can be parametrized in terms of the Euclidean geometry of $\Omega_{g d h}$ and $\Omega_{g^{-1}} d h$. These infinitesimal motions are given by infinitesimal changes in lengths of finite sides with the changes being done simultaneously on $\Omega_{g d h}$ and $\Omega_{g^{-1}}$ th to preserve conjugacy of the periods. The link to the conformal geometry is that a motion which infinitesimally transforms $\Omega_{g d h}$, say, will produce an infinitesimal change in the conformal structure. Tensorially, this tangent vector to the moduli space of conformal structures is represented by a Beltrami differential.

3.3.1. Infinitesimal pushes. The goal of this subsection is to explicitly compute the effect of infinitesimal pushes of certain edges in an orthodisk on the conformal geometry of that orthodisk. This is done by explicitly displaying the infinitesimal deformation of that orthodisk as a map of the plane to itself and then computing the Beltrami differential of that map. An important point is that these computations result in explicit formulas.

In what follows, we rotate our picture by an angle of $\pi / 4$ so that all of our boundary edges are either horizontal or vertical. This simplifies our notation somewhat, even if it complicates the meaning of "conjugacy".

We first consider the case of a horizontal finite side, say E; as in Figure 3, we see that the neighborhood of the horizontal side of the orthodisk in the plane naturally divides into six regions which we label $R_{1}, \ldots, R_{6}$. Our deformation $f_{\varepsilon}=f_{\varepsilon, b, \delta}$ differs from the identity only in such a neighborhood, and in each of the six regions, the map is affine. In fact, we have a two-parameter family of these deformations, all of which have the same infinitesimal effect, with the parameters $b$ and $\delta$ depending 


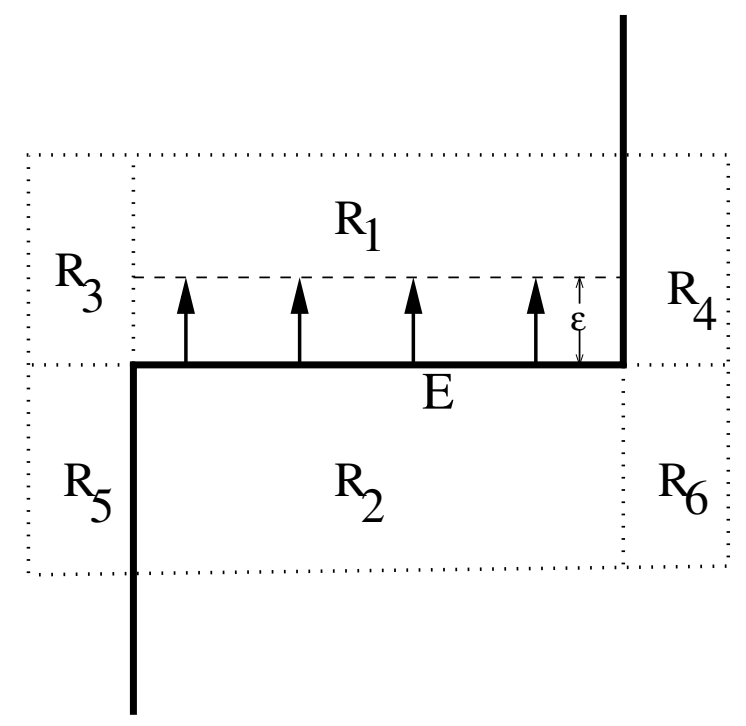

FiguRE 3. Domain for Beltrami differential computation

on the dimensions of the supporting neighborhood

(6)

$$
f_{\varepsilon}(x, y)= \begin{cases}\left(x, \varepsilon+\frac{b-\varepsilon}{b} y\right), & \{-a \leq x \leq a, 0 \leq y \leq b\}=R_{1} \\ \left(x, \varepsilon+\frac{b+\varepsilon}{b} y\right), & \{-a \leq x \leq a,-b \leq y \leq 0\}=R_{2} \\ \left(x, y+\frac{\varepsilon+\frac{b-\varepsilon}{b} y-y}{\delta}(x+\delta+a)\right), & \{-a-\delta \leq x \leq-a, 0 \leq y \leq b\}=R_{3} \\ \left(x, y-\frac{\varepsilon+\frac{b-\varepsilon}{b} y-y}{\delta}(x-\delta-a)\right), & \{a \leq x \leq a+\delta, 0 \leq y \leq b\}=R_{4} \\ \left(x, y+\frac{\varepsilon+\frac{b+\varepsilon}{b} y-y}{\delta}(x+\delta+a)\right), & \{-a-\delta \leq x \leq-a,-b \leq y \leq 0\}=R_{5} \\ \left(x, y-\frac{\varepsilon+\frac{b+\varepsilon}{b} y-y}{\delta}(x-\delta-a)\right), & \{a \leq x \leq a+\delta,-b \leq y \leq 0\}=R_{6} \\ (x, y) & \text { otherwise, }\end{cases}
$$

where we have defined the regions $R_{1}, \ldots, R_{6}$ within the definition of $f_{\varepsilon}$. Also, note that here the orthodisk contains the arc $\{(-a, y) \mid 0 \leq y \leq b\} \cup\{(x, 0) \mid-a \leq x \leq$ $a\} \cup\{(a, y) \mid-b \leq y \leq 0\}$. Let $E$ denote the edge being pushed, defined above as $[-a, a] \times\{0\}$.

Let $\nu_{\varepsilon}=\frac{\left(f_{\varepsilon}\right)_{\bar{z}}}{\left(f_{\epsilon}\right)_{z}}$ denote the Beltrami differential of $f_{\varepsilon}$, and set $\dot{\nu}=\left.\frac{d}{d \varepsilon}\right|_{\varepsilon=0} \nu_{\epsilon}$. It is easy to compute that $\dot{\nu}=\left[\left.\frac{d}{d \varepsilon}\right|_{\varepsilon=0}\left(f_{\varepsilon}\right)\right]_{\bar{z}}$ evaluates near $E$ to be

(7)

$$
\dot{\nu}= \begin{cases}\frac{1}{2 b}, & z \in R_{1} \\ -\frac{1}{2 b}, & z \in R_{2} \\ \frac{1}{2 b}[x+\delta+a] / \delta+i\left(1-\frac{y}{b}\right) \frac{1}{2 \delta}=\frac{1}{2 b \delta}(\bar{z}+\delta+a+i b), & z \in R_{3} \\ -\frac{1}{2 b}[x-\delta-a] / \delta-i\left(1-\frac{y}{b}\right) \frac{1}{2 \delta}=\frac{1}{2 b \delta}(-\bar{z}+\delta+a-i b), & z \in R_{4} \\ -\frac{1}{2 b}[x+\delta+a] / \delta+i\left(1+\frac{y}{b}\right) \frac{1}{2 \delta}=\frac{1}{2 b \delta}(-\bar{z}-\delta-a+i b), & z \in R_{5} \\ \frac{1}{2 b}[x-\delta-a] / \delta-i\left(1+\frac{y}{b}\right) \frac{1}{2 \delta}=\frac{1}{2 b \delta}(\bar{z}-\delta-a-i b), & z \in R_{6} \\ 0 & z \notin \operatorname{supp}\left(f_{\varepsilon}-\mathrm{Id}\right) .\end{cases}
$$


3.3.2. Deformation of a conjugate pair. With these definitions in place, we prove that a conformal pair $\left(\Omega_{g d h}, \Omega_{g^{-1} d h}\right)$ of conjugate orthodisks (recall that this refers to a pair of domains with zigzag boundaries composed of conjugate edge vectors) admits only trivial deformations. Let the $\Delta_{\varkappa}$ denote the space of conjugate pairs of orthodisks of the type described at the opening of the argument in subsection 3.3 . The two-fold branched cover of the double of one of these orthodisks is, after quotient by a translation, a surface of genus $\varkappa$ (which branches over the sphere at a set of $2 \varkappa+2$ points on an equator). Thus, we may regard $\Delta_{\varkappa}$ as a subset $\Delta_{\varkappa} \subset \mathcal{T}_{\varkappa} \times \mathcal{T}_{\varkappa}$ of the product of a pair of Teichmüller spaces $\mathcal{T}_{\varkappa}$; note $\operatorname{dim} \Delta_{\varkappa}=\operatorname{dim} \mathcal{T}_{\varkappa}$. Let $X_{0}$ denote a pair which is related by a conformal map $\zeta: \Omega_{g d h} \rightarrow \Omega_{g^{-1} d h}$ (and which therefore defines a periodic minimal surface with the appropriate ends); note that such an example may be regarded as an element $X_{0} \in \Delta_{\varkappa} \subset \mathcal{T}_{\varkappa} \times \mathcal{T}_{\varkappa}$.

We claim that $\Delta_{\varkappa}$ is transverse to the diagonal $D_{\varkappa}=\{(\mathcal{R}, \mathcal{R})\} \subset \mathcal{T}_{\varkappa} \times \mathcal{T}_{\varkappa}$, where of course $\operatorname{dim} D_{\varkappa}=\operatorname{dim} \Delta_{\varkappa}$. To see this, note that we may regard a tangent direction as a pair $\left(\dot{\nu}_{g d h}, \dot{\nu}_{g^{-1} d h}\right)$ of Beltrami differentials, each representing a tangent direction to the points $\left[\Omega_{g d h}\right] \in \mathcal{T}_{\varkappa}$ and $\left[\Omega_{g^{-1} d h}\right] \in \mathcal{T}_{\varkappa}$, respectively. Yet at $X_{0}$, the points $\left[\Omega_{g d h}\right]$ and $\left[\Omega_{g^{-1} d h}\right]$ represent the identical point in $\mathcal{T}_{\varkappa}$, so we begin by computing how the Beltrami differentials $\dot{\nu}_{g d h}$ and $\dot{\nu}_{g^{-1} d h}$ relate to one another. To this end, consider how an infinitesimal push in the sense of the previous section on an edge $E$ defines Beltrami differentials $\dot{\nu}_{g d h}$ and $\dot{\nu}_{g^{-1}} d h$. Of course, the conjugacy of $\Omega_{g d h}$ and $\Omega_{g^{-1} d h}$ provides, via the formulas of that section, the basic defining relation that if $\dot{\nu}_{g d h}$ has local expansion $\dot{\nu}_{g d h}(z)=\frac{1}{2 b} \frac{d \bar{z}}{d z}$ near an interior point of an edge $E$, then also $\dot{\nu}_{g^{-1} d h}(\zeta)=\frac{1}{2 b} \frac{d \bar{\zeta}}{d \zeta}$ at the corresponding edge. However, since $X_{0}$ is a pair of conformally related orthodisks, we may also assume, in this particular case, the existence of a conformal map $\zeta: \Omega_{g d h} \longrightarrow \Omega_{g^{-1} d h}$ which preserves the vertices. Such a map takes vertical sides to horizontal sides by construction and this has the local expansion $\zeta= \pm i|c| z+O\left(|z|^{2}\right)$ near an interior point of an edge. We therefore compute the pullback of $\dot{\nu}_{g^{-1}} d h$ to $\Omega_{g d h}$ as

$$
\begin{aligned}
\dot{\nu}_{g^{-1} d h}(\zeta) \frac{d \bar{\zeta}}{d \zeta} & =\dot{\nu}_{g^{-1} d h}(\zeta) \frac{\bar{\zeta}^{\prime}}{\zeta^{\prime}} \frac{d \bar{z}}{d z} \\
& =\left(-\dot{\nu}_{g^{-1} d h}(\zeta)+\text { h.o.t. }\right) d \bar{z} / d z \\
& =-\frac{1}{2 b} \frac{d \bar{z}}{d z}
\end{aligned}
$$

along the edge $E$. We conclude that, locally in the interior of the edge $E$, we have $\zeta^{*} \dot{\nu}_{g^{-1} d h}=-\dot{\nu}_{g d h}+o_{b}(1)$, where $o_{b}(1)$ may be taken arbitrarily small. The above computation nearly proves that $\left[\dot{\nu}_{g d h}\right]=-\left[\dot{\nu}_{g^{-1} d h}\right]$ as elements of the tangent space $T_{\Omega_{g d h}} \mathcal{T}_{\varkappa}=T_{\Omega_{g^{-1} d h}} \mathcal{T}_{\varkappa}$ : what is left to prove for that conclusion is that the contributions to $\left[\dot{\nu}_{g d h}\right]$ or $\left[\dot{\nu}_{g^{-1} d h}\right]$ from the regions $R_{3}$ and $R_{5}$ - and their counterparts in the other fundamental domains for the $\mathbf{Z}_{2} \oplus \mathbf{Z}_{2}$ group of Alexandrov reflections - are negligible. To see this, begin by noticing that our surface $\bar{M}$ is hyperelliptic, branching over the points where the Gauss map $g$ is singular, i.e., over the images of the endpoints of the edges of the zigzags. Recall next that the pairing of the tangent and cotangent spaces to $\mathcal{T}_{\varkappa}$ is given by integrating the product of Beltrami differentials and holomorphic quadratic differentials on $\bar{M}$. Now, as $\bar{M}$ is hyperelliptic, the Taylor expansion of a holomorphic quadratic differential $\Phi$ on $\bar{M}$ around (the lift of) a corner of an edge is even, i.e., $\Phi=\left(c_{0}+c_{2} w^{2}+\right.$ h.o.t.) $d w^{2}$. 
This descends to a neighborhood of a corner of the edge via the local map $w=z^{\frac{1}{3}}$ as $\Phi=\left(\frac{1}{9} c_{0} z^{-\frac{4}{3}}+\frac{1}{9} c_{2} z^{-\frac{2}{3}}+O_{z}(1)\right) d z^{2}$.

In terms of this expression, the terms of order $O\left(z^{-\frac{2}{3}}\right)$ and higher are easily seen to integrate against $\left[\dot{\nu}_{g d h}\right]$ or $\left[\dot{\nu}_{g^{-1} d h}\right]$ to negligible amounts in a small neighborhood of the endpoints of an edge, but the term of order $O\left(z^{-\frac{4}{3}}\right)$ is more worrisome. To understand that it also has but a negligible effect, we need to consider its effect in an entire neighborhood in $\bar{M}$ of an endpoint of an edge of the zigzag. In particular, we consider the development of the other three fundamental domains whose closures contain that point: these developments are obtained by reflection across the edges incident to that point. After a reflection in each of these edges, we obtain the development of the domain in the image of $w \mapsto-w$ of our original domain: it has the form in Figure 3 consisting of the regions $R_{2}, R_{4}$ and $R_{6}$. In that region, because the deformation of the relevant edge will be downwards instead of upwards, the Beltrami differentials $\dot{\nu}_{g^{-1} d h}$ and $\dot{\nu}_{g d h}$ should be regarded as expressed by the formulas for $-\dot{\nu}$ in (7). In terms of these explicit formulas and using that the map between these developments may be written as $\Psi: z \mapsto-z$ (abusing notation by setting $a=0$ in both formulas), we note that

$$
\Psi^{*}\left(-\left.\dot{\nu}\right|_{R_{6}}\right)+\left.\dot{\nu}\right|_{R_{3}}=\Psi^{*}\left(-\left.\dot{\nu}\right|_{R_{4}}\right)+\left.\dot{\nu}\right|_{R_{5}}=0 .
$$

On the other hand, since $\Phi$ is even, we have that $\Psi^{*} \Phi=\Phi$, and so we conclude that the pairing $\int \Phi \dot{\nu}$ between $\Phi$ and either $\dot{\nu}=\dot{\nu}_{g^{-1}} d h$ or $\dot{\nu}=\dot{\nu}_{g d h}$ over the images of the regions $R_{3}, R_{4}, R_{5}$ and $R_{6}$ (in all of the fundamental domains) vanishes: the effect of $\dot{\nu}_{g^{-1}} d h$ and $\dot{\nu}_{g d h}$ as tangent vectors in a pairing with covectors is given by integration over (the images of) the regions $R_{1}$ (and $R_{2}$ ).

We conclude that for $\dot{\nu}_{g^{-1} d h}$ and $\dot{\nu}_{g d h}$ defining an infinitesimal push, we have $\left[\zeta^{*} \dot{\nu}_{g^{-1} d h}\right]=-\left[\dot{\nu}_{g d h}\right]$ as elements of the tangent space to $\mathcal{T}_{\varkappa}$. Furthermore, since any deformation of $X_{0}$ is given by a linear combination of such infinitesimal pushes, we conclude that $\left[\dot{\nu}_{g d h}\right]=-\left[\dot{\nu}_{g^{-1} d h}\right]$ as elements of the tangent space $T_{\Omega_{g d h}} \mathcal{T}_{\varkappa}=$ $T_{\Omega_{g^{-1} d h}} \mathcal{T}_{\varkappa}$. Thus, any conjugacy-preserving deformation of $X_{0}$ destroys the conformal equivalence of $\Omega_{g d h}$ and $\Omega_{g^{-1} d h}$ to the order of the deformation, a statement which implies that $\Delta_{\varkappa}$ is transverse to the diagonal. This concludes the proof of the claim.

To finish the proofs of Proposition 4 and Lemma 5 observe that any deformation $M_{t}$ of minimal surfaces through $M_{0}$ must preserve the conformality between $\Omega_{g d h}(t)$ and $\Omega_{g^{-1} d h}(t)$. Thus, by the computation above, we conclude that the tangent vector for $M_{t}$ is trivial as a tangent vector to Teichmüller space, and moreover, the forms $g d h$ and $\frac{1}{g} d h$ are only trivially deformed. Since these forms suffice as Weierstraß data, we conclude that $M_{t}$ deforms only by an infinitesimal congruence, as desired.

Recall the moduli spaces $\mathcal{M}(\theta)$ of pairs of surfaces $\left(\Omega_{g d h}, \Omega_{g^{-1} d h}\right)$ whose zigzag boundaries have orbits of points which ascend at slope $m(\theta)$ under the translation group. The paragraph above shows that $\mathcal{M}\left(\theta_{0}\right)$ meets the diagonal $D_{\varkappa}$ in $\mathcal{T}_{\varkappa} \times \mathcal{T}_{\varkappa}$ transversely. As $\operatorname{dim}(\mathcal{M}(\theta))+\operatorname{dim}\left(\mathcal{T}_{\varkappa}\right)=\operatorname{dim}\left(\mathcal{T}_{\varkappa} \times \mathcal{T}_{\varkappa}\right)$, the implicit function theorem implies that there is a curve $X_{\theta} \in \mathcal{M}(\theta) \cap D_{\varkappa}$ for $\theta$ near $\theta_{0}$. By our construction of zigzags, such an element $X_{\theta}$ represents a periodic minimal surface whose ends make an angle $\theta$ with each other. This concludes the proofs of Proposition 4 and Lemma 5 , 


\section{Properness of the ANGLE MAP}

In this section, we prove the following properness result.

Proposition 7. The angle map $\theta: \mathcal{M}_{\varkappa} \rightarrow\left(0, \frac{\pi}{2}\right]$ is proper.

Proof. Since $\mathcal{M}_{\varkappa}$ consists of curves and $\theta: \mathcal{M}_{\varkappa} \rightarrow\left(0, \frac{\pi}{2}\right]$ is a local diffeomorphism by Proposition 4, it is sufficient to prove that if $\left\{\bar{M}_{n}\right\}_{n \in \mathbb{N}}$ is a sequence of examples in $\mathcal{M}_{\varkappa}$ with $\lim _{n \rightarrow \infty} \theta\left(\bar{M}_{n}\right)=\theta_{0}>0$, then a subsequence of the surfaces converges on compact subsets of $\mathbb{E}^{3} / \mathbb{Z}$ to a minimal surface $\bar{M}_{\infty} \in \mathcal{M}_{\varkappa}$ with $\theta\left(\bar{M}_{\infty}\right)=\theta_{0}$. Note that we choose the surfaces $\left\{\bar{M}_{n}\right\}_{n \in \mathbb{N}}$ so that each lies in $\mathbb{E}^{3} / \mathbb{Z}$ and is invariant under reflection in the vertical annuli $A_{x_{1}}, A_{x_{2}}$ in $\mathbb{E}^{3} / \mathbb{Z}$ given in the statement of Proposition 3

As the four ends of $\bar{M}_{n}$ are asymptotically flat, the lifted surface $M_{n}$ in $\mathbb{E}^{3}$ has area growth constant $A\left(M_{n}\right)=2 \pi$. Thus, by the monotonicity formula for area of a minimal surface in $\mathbb{E}^{3}$, every $M_{n}$ has at most $2 \pi r^{2}$ of area in extrinsic balls of radius $r$. Hence, after choosing $r<\frac{1}{2}$ so that the balls in $\mathbb{E}^{3}$ embed in the quotient space, we see that the surfaces $\bar{M}_{n}$ have locally bounded area in $\mathbb{E}^{3} / \mathbb{Z}$. We now check that there are also uniform estimates for the curvature of the surfaces in the sequence.

Arguing by contradiction and after extracting a subsequence, suppose that there exist points $p_{n} \in \bar{M}_{n}$ with maximal absolute curvature $\lambda_{n}^{2} \geq n$; note that maximal curvature points $p_{n}$ exist since the asymptotic curvature of the $\bar{M}_{n}$ is zero. Consider the homothetically expanded surfaces $\widetilde{M}_{n}=\lambda_{n}\left[\bar{M}_{n} \cap B\left(p_{n}, \frac{1}{2}\right)\right]$ in the homothetically expanded ball $\lambda_{n} B\left(p_{n}, \frac{1}{2}\right)$. The surfaces $\widetilde{M}_{n}$ are submanifolds in the flat three-manifolds $\lambda_{n} B\left(p_{n}, \frac{1}{2}\right)$, which are isometric to balls $B\left(\overrightarrow{0}, r_{n}\right)$ of radius $r_{n}=\frac{\lambda_{n}}{2} \geq \frac{\sqrt{n}}{2}$ in $\mathbb{E}^{3}$ centered at the origin $\overrightarrow{0}$; these balls are converging naturally to all of $\mathbb{E}^{3}$. Consider the surfaces $\widetilde{M}_{n}$ to lie in $B\left(\overrightarrow{0}, r_{n}\right) \subset \mathbb{E}^{3}$. Since the $\widetilde{M}_{n}$ have maximal absolute curvature 1 at the origin and in balls of radius $r \leq r_{n}$ have area at most $2 \pi r^{2}$, standard results (see, for example, 8]) imply that a subsequence of these surfaces converges on compact subsets of $\mathbb{E}^{3}$ to a properly embedded minimal surface $\widetilde{M}$ in $\mathbb{E}^{3}$ with absolute curvature at most 1 and with absolute curvature 1 at the origin. The surface $\widetilde{M}$ is connected by the strong half-space theorem 1]. Furthermore, since each surface $\widetilde{M}_{n}$ has the same total absolute curvature as $\bar{M}_{n} \cap B\left(p_{n}, \frac{1}{2}\right)$, then each $\widetilde{M}_{n}$ has total absolute curvature less than the total absolute curvature of $\bar{M}_{n}$, which by the Gauss-Bonnet formula is $-2 \pi \chi\left(\bar{M}_{n}\right)$, which is the finite number $4 \pi(\varkappa+1)$. In particular, $\widetilde{M}$ has finite total curvature. Since $\widetilde{M}$ is embedded and not flat, it has at least two ends which are asymptotic to either planes or ends of catenoids. Since $\widetilde{M}$ has area growth constant at most $2 \pi$ and it is not a plane, then it has exactly two ends. By Schoen's theorem [16, we see that $\widetilde{M}$ is a catenoid with waist circle passing through the origin.

We claim that each of the Alexandrov annuli of symmetry of the $\bar{M}_{n}$ intersect $B\left(p_{n}, r_{n}\right)$ for $n$ large and limit to annuli of symmetry for $\widetilde{M}$. Otherwise, the surface $\widetilde{M}$ would be the limit of domains in $\widetilde{M}_{n} \subset \lambda_{n} \bar{M}_{n}$, which are graphical over their projections to one of its Alexandrov annuli of symmetry. It would then follow that the Gaussian image of $\widetilde{M}$ would lie in a hemisphere of $\mathbb{S}^{2}$, which is false for a catenoid. Hence, the Alexandrov annuli of symmetry of the $\widetilde{M}_{n}$ limit in a natural way to Alexandrov planes of symmetry of $\widetilde{M}$, which, after a translation of $\widetilde{M}$, 
we can consider to be the $\left(x_{1}, x_{3}\right)$ - and $\left(x_{2}, x_{3}\right)$-planes. Furthermore, since each component of the fixed point set of an Alexandrov annulus of symmetry is a simple closed convex planar curve, which is invariant under reflection across the other such Alexandrov annulus and hence has two fixed points, then it is easy to show that, since $\widetilde{M}$ is connected, the fixed point set of one of the planes of Alexandrov symmetry of $\widetilde{M}$ must intersect the fixed point set of the other plane of symmetry of $\widetilde{M}$. It follows that $\widetilde{M}$ is a catenoid with axis being either the $x_{1}$-axis or the $x_{2}$-axis (rather than with axis being the $x_{3}$-axis).

From the discussion in the previous paragraph, we conclude that a subsequence of the locally finite integral minimal varifolds $\bar{M}_{n}$ in $\mathbb{E}^{3} / \mathbb{Z}$ converges to a limit minimal varifold $\bar{M}_{\infty}$ with mass density 2 at some point of the vertical circle $\alpha=(0,0) \times \mathbb{R} / \mathbb{Z}$ in $\mathbb{E}^{3} / \mathbb{Z}$. It follows from the monotonicity formula for area that $\bar{M}_{\infty}$ is the union of two flat vertical annuli, not necessarily distinct and both containing $\alpha$.

We claim that the convergence of the $\bar{M}_{n}$ to $\bar{M}_{\infty}$ is smooth away from $\alpha$. If not, then there exists a point $p \in \mathbb{E}^{3} / \mathbb{Z}-\alpha$, such that, after extracting a subsequence, the absolute curvature of the $\bar{M}_{n}$ in the $\varepsilon=\frac{1}{2} d(p, \alpha)$ ball $B(p, \varepsilon)$ centered at $p$ is at least $n$. Let $q_{n}$ be a point of $\widehat{M}_{n}=\bar{M}_{n} \cap B(p, \varepsilon)$, where the function $d\left(\cdot, \partial \widehat{M}_{n}\right)|K|(\cdot)$ has its maximum value; here, $|K|(\cdot)$ is the absolute curvature function on $\widehat{M}_{n}$. Let $\lambda_{n}=\sqrt{\left|K\left(q_{n}\right)\right|}$ and note that the surfaces $\Sigma_{n}=\lambda_{n}\left(\widehat{M}_{n} \cap B\left(q_{n}, r_{n}\right)\right)$, where $r_{n}=\frac{1}{2} d\left(q_{n}, \partial B(p, \varepsilon)\right)$, have bounded curvature in the balls $\lambda_{n} B\left(q_{n}, r_{n}\right)$ of radius $r_{n}$ centered at $q_{n}$. These metric balls converge to $\mathbb{E}^{3}$ with $q_{n}$ considered to be at the origin. The surfaces $\Sigma_{n}$ have absolute curvature bounded by 4 and have local area estimates. Our previous arguments now imply that a subsequence of the $\Sigma_{n}$ converges to a catenoid in $\mathbb{E}^{3}$. Since the Gauss map of this catenoid is essentially onto, it contains a point which is a limit of points coming originally from $\alpha=A_{x_{1}} \cap A_{x_{2}}$. This is a contradiction, since $\alpha$ is disjoint from $B(p, \varepsilon)$. This contradiction proves that the surfaces $\bar{M}_{n}$ converge smoothly with multiplicity two to $\bar{M}_{\infty}$, away from $\alpha$.

We claim that $\bar{M}_{\infty}$ is either $A_{x_{1}}$ or $A_{x_{2}}$ with multiplicity two. If not, then since $\bar{M}_{\infty}$ is the union of two flat vertical annuli, the circle $\alpha \subset \bar{M}_{\infty}$ is contained in the intersection set of two distinct vertical flat annuli, and so, every point $p \in \alpha$ is a point in the singular set of convergence to $\bar{M}_{\infty}$. Now, the blow-up argument in the previous paragraph shows that, for any $\varepsilon>0$ the ball $B(p, \varepsilon)$ contains for $n$ large, an approximately scaled down catenoid in $\bar{M}_{n}$, and so, the total absolute curvature of $\bar{M}_{n} \cap B(p, \varepsilon)$ is at least $3 \pi$ for $n$ large. Since $\varepsilon$ is arbitrary and the total absolute curvature of $\bar{M}_{n}$ is $4 \pi(\varkappa+1)$, which is finite, we obtain a contradiction. Hence, $\bar{M}_{\infty}$ is $A_{x_{1}}$ or $A_{x_{2}}$ with multiplicity two as a limit varifold. (Although we do not use it here, we observe that this argument also shows, after choosing a subsequence, that there are at most $\varkappa+1$ distinct singular points of convergence of the $\bar{M}_{n}$ to $A_{x_{1}}$ or $A_{x_{2}}$, which by our earlier arguments must lie on $\alpha$.)

Let $N_{\varepsilon}(\alpha)$ be any fixed $\varepsilon>0$ neighborhood around $\alpha$. For $n$ large, our analysis of the limits $\lambda_{n} \bar{M}_{n}$ shows that $M_{n}-N_{\varepsilon}(\alpha)$ consists of four annular Scherk ends of $M_{n}$. Now, we have also shown that the convergence of $\bar{M}_{n}-N_{\varepsilon}(\alpha)$ to $\bar{M}_{\infty}-N_{\varepsilon}(\alpha)$ is smooth along $\partial\left(\bar{M}_{n}-N_{\varepsilon}(\alpha)\right)$, so the flux vectors (integrals of the conormals along each component of $\left.\partial\left(\bar{M}_{n}-N_{\varepsilon}(\alpha)\right)\right)$ are converging to either $( \pm 1,0,0)$ or $(0, \pm 1,0)$, since those limits are the flux vectors of $\bar{M}_{\infty}$. But these flux vectors are also the flux vectors of the ends of $\bar{M}_{n}$ by the divergence theorem, and the flux vectors of 
the ends of $\bar{M}_{n}$ are bounded away from $( \pm 1,0,0)$, since the limit angle $\theta_{0}>0$. This contradiction implies that the sequence $\left\{\bar{M}_{n}\right\}_{n \in \mathbb{N}}$ with $\theta\left(\bar{M}_{n}\right) \rightarrow \theta_{0}>0$ has uniformly bounded curvature.

We now prove that our original sequence $\left\{\bar{M}_{n}\right\}_{n \in \mathbb{N}}$ with $\theta\left(\bar{M}_{n}\right) \rightarrow \theta_{0}>0$ converges to an example $\bar{M}_{\infty} \in \mathcal{M}_{\varkappa}$. Since the sequence of surfaces has uniformly bounded curvature and local area estimates, a subsequence converges on compact subsets of $\mathbb{E}^{3} / \mathbb{Z}$ to a properly embedded minimal surface $\bar{M}_{\infty}$. Recall that the sum of the lengths of the convex curves in $M_{n} \cap A_{x_{i}}$ corresponds to the flux of $\nabla x_{(i+1)} \bmod 2$ of $M_{n}$, which is less than or equal to 2 and is determined by $\theta\left(M_{n}\right)$. Since every convex curve in $\bar{M}_{n} \cap\left(A_{x_{1}} \cup A_{x_{2}}\right)$ intersects the vertical circle $\alpha$, the $\varkappa+1$ convex curves $\bar{M}_{n} \cap A_{x_{i}}$ converge smoothly to $\varkappa+1$ convex curves in $\bar{M}_{\infty} \cap A_{x_{i}}$, each of length less than 2 for $i=1,2$. Moreover, the lengths of these curves are also bounded away from zero, since they are planar curves and principal on $M_{n}$ : any pinching of them would then blow up the curvature somewhere along them. Yet these lengths correspond to the lengths of the segments in the zigzags bounding the domains $\Omega_{g d h}$ and $\Omega_{g^{-1} d h}$ for $M_{n}$, so we see that these domains $\Omega_{g d h}$ and $\Omega_{g^{-1}} d h$ for $M_{n}$ also converge smoothly and without degeneration to the orthodisks of $\bar{M}_{\infty}$.

These orthodisks, together with the implied vertex-preserving conformal map between them, of course determine the Weierstraß data for a minimal surface whose geometry is given by the Euclidean geometry of the orthodisks. Here, since the limiting orthodisk has a fundamental domain bounded by $2 \varkappa+2$ nondegenerate segments of alternating slope, we see that the surface $\bar{M}_{\infty}$ is a nondegenerate minimal surface of genus $\varkappa$. Furthermore, as the flux is determined (see Proposition 6 ) by the slope of the orbit of a vertex, and the orthodisks are converging smoothly, we see that $\theta\left(\bar{M}_{\infty}\right)=\theta_{0}$.

This completes the proof of the proposition.

Remark 8. We note that the flux argument given in the curvature estimate part of the proof of Proposition 7 implies that if, for some sequence in $\left\{\bar{M}_{n}\right\}_{n \in \mathbb{N}}$ in $\mathcal{M}_{\varkappa}$ we have $\theta\left(\bar{M}_{n}\right) \rightarrow 0$, then the locally finite limit integral minimal varifold $\bar{M}_{\infty}$ is the annulus $A_{x_{1}}$ with multiplicity two.

\section{Small Angle examples are Scherk EXAmples}

In this section, we prove the following result.

Proposition 9. For every $\varkappa>0$, there exists an $\varepsilon>0$ such that if $\bar{M} \in \mathcal{M}_{\varkappa}$ and $\theta(\bar{M})<\varepsilon$, then $\bar{M}$ is a Scherk example.

Proof. Suppose $\bar{M}_{n} \in \mathcal{M}_{\varkappa}$ is a sequence of examples, where $\theta\left(\bar{M}_{n}\right)<\frac{1}{n}$. After extracting a subsequence, the sequence $\bar{M}_{n}$ converges to an integral varifold $\bar{M}_{\infty}$. From the proof of Proposition 7 and Remark 8, it is easy to see that $\bar{M}_{\infty}$ is one of the annuli $A_{x_{1}}$ or $A_{x_{2}}$ (with multiplicity two) of symmetry and that the limiting flux vectors to the ends of the $\bar{M}_{n}$ converge to vectors in $\bar{M}_{\infty}$. Hence, $\bar{M}_{\infty}$ corresponds to $A_{x_{1}}$. Modifications of the arguments used in the proof of Proposition 7 also show that, for $\alpha=(0,0) \times \mathbb{R} / \mathbb{Z}$ and $n$ large, there exist $\varkappa+1$ points $P_{n}=\left\{p_{1}(n), p_{2}(n), \ldots, p_{\varkappa+1}(n)\right\} \subset \bar{M}_{n} \cap \alpha$ with normal vector $(0,0,1)$ together with small positive numbers $\varepsilon_{1}(n), \varepsilon_{2}(n), \ldots, \varepsilon_{\varkappa+1}(n)$, such that for each $i$, the intersection $B\left(p_{i}(n), \varepsilon_{i}(n)\right) \cap \bar{M}_{n}$ is a compact annulus which is $C^{2}$-close to a standard catenoid with axis along the $x_{2}$-axis, which has been scaled by the inverse 
of the square root of the absolute curvature at $p_{i}(n)$. Furthermore, after replacing by a subsequence, the surface $\widetilde{M}_{n}=\bar{M}_{n} \cap\left[\mathbb{E}^{3} / \mathbb{Z}-\bigcup_{i=1}^{\varkappa+1} B\left(p_{i}(n), \varepsilon_{i}(n)\right)\right]$ consists of two components which are graphs of gradient less than $\frac{1}{n}$ over their projections to the annulus $\bar{M}_{\infty}$.

A subsequence of the (paired) graphs $\widetilde{M}_{n}$ converges smoothly to $\bar{M}_{\infty}$ punctured in at most $\varkappa+1$ points with graphical gradients converging to zero as $n \rightarrow \infty$. Thus, the degenerating conformal structures of $\bar{M}_{n}$ as $n \rightarrow \infty$ can be seen to converge to that of two copies of the annulus $A_{x_{1}}$ (parallel to the $x_{1}$-axis) with nodes forming at the (limits of the) $\varkappa+1$ points $P_{n}$ along $\alpha \subset \bar{M}_{\infty}$.

Now let $\widehat{A}_{n}$ denote the $\mathbb{Z}$-cover of one of the four fundamental domain annuli $A_{n}$ of $M_{n}$ and let $\widehat{A}$ denote the limit of the $\widehat{A}_{n}$. Thus, the domains $\widehat{A}_{n}$ and $\widehat{A}$ are conformal half-planes, with the $(\varkappa+1)$-tuple of points $P_{n}$ lifting to a periodic sequence $\widehat{P}_{n}$ of boundary points of $\widehat{A}_{n}$; the sequence $\widehat{P}_{n}$ converges to a periodic sequence $\widehat{N}_{n}$ of lifts of the nodes $N_{n}$ on the boundary of $\widehat{A}$. We shall also have need of the periodic sequence $\widehat{Q}_{n} \subset \partial \widehat{A}_{n}$ which are lifts of the points $Q_{n} \in \bar{M}_{n} \cap \alpha$ whose normal vectors are $(0,0,-1)$. Naturally, the points $\widehat{P}_{n}$ and $\widehat{Q}_{n}$ alternate in position along $\partial \widehat{A}_{n}$ and $\widehat{Q}_{n} \rightarrow \widehat{N}_{n}$ along with $\widehat{P}_{n}$. We take the upper halfplane $\mathbf{H}$ as a model for $\widehat{A}_{n}$ and $\widehat{A}$, and we let the images of $\widehat{P}_{n}$ be given by $\left\{a_{k, \ell}=k+a_{\ell} \mid \ell=0, \ldots, \varkappa\right.$ where $a_{0}=0$ and $0<a_{\ell}<1$ for $\left.\ell>1\right\}$ and the images of $\widehat{Q}_{n}$ be given by $\left\{b_{k, \ell}=k+b_{\ell} \mid \ell=0, \ldots, \varkappa\right.$, where $\left.0<b_{\ell}<1\right\}$. Naturally we take $a_{k, j}<b_{k, j}<a_{k, j+1}<b_{k, j+1}$ for every $k$. These points $a_{k, j}$ and $b_{k, j}$ depend on $n$, but we will suppress the natural dependence on $n$ until it is relevant and important.

As in section 3, we consider the forms $g d h$ and $\frac{1}{g} d h$ on $\widehat{A}_{n}$ and $A_{n}$. These evidently develop to domains bounded by a periodic boundary, as described in section 3.1. We observe that we may parametrize the domains $\Omega_{g d h}$ and $\Omega_{g^{-1} d h}$ via the Schwarz-Christoffel maps (suppressing the dependence on $n$ )

$$
F_{\Omega_{g d h}}(\zeta)=e^{i \pi / 4} K_{+} \int^{\zeta} \prod_{j=0}^{\varkappa}\left[\frac{z-a_{0, j}}{z-b_{0, j}}\right]^{1 / 2} \prod_{k=1}^{\infty} \prod_{j=0}^{\varkappa}\left[\frac{\left(z-a_{k, j}\right)\left(z-a_{-k, j}\right)}{\left(z-b_{k, j}\right)\left(z-b_{-k, j}\right)}\right]^{1 / 2} d z
$$

and

$F_{\Omega_{g^{-1}} d h}(\zeta)=e^{-i \pi / 4} K_{-} \int^{\zeta} \prod_{z=0}^{\varkappa}\left[\frac{z-b_{0, j}}{z-a_{0, j}}\right]^{1 / 2} \prod_{k=1}^{\infty} \prod_{j=0}^{\varkappa}\left[\frac{\left(z-b_{k, j}\right)\left(z-b_{-k, j}\right)}{\left(z-a_{k, j}\right)\left(z-a_{-k, j}\right)}\right]^{1 / 2} d z$.

To see this, first observe that as the terms $\left(\frac{\left(z-a_{k, j}\right)\left(z-a_{-k, j}\right)}{\left(z-b_{k, j}\right)\left(z-b_{-k, j}\right)}\right)^{1 / 2}$ are asymptotically $1+O\left(\frac{1}{k^{2}}\right)$ for $k$ large, the infinite product converges absolutely, and uniformly on compacta in $\overline{\mathbf{H}}$. Moreover, the images of the boundary are evidently periodic zigzags: they are zigzags by the basic Schwarz-Christoffel theory, and they are periodic as the periodicity of the coefficients $\left(a_{k+1, j}=a_{k, j}+1, b_{k+1, j}=b_{k, j}+1\right)$ forces the periodicity of the developing maps $F_{\Omega_{g d h}}$ and $F_{\Omega_{g^{-1} d h}}$. The factors $K_{+}$ and $K_{-}$normalize the size of the images; of course, by scaling, we can set $K_{+}=1$, leaving us to estimate only $K_{-}$.

Now, the crucial part of the analysis is the determination of the coefficients $a_{k, j}$ and $b_{k, j}$ : we know that as $n \rightarrow \infty$, we have $\left|a_{k, j}-b_{k, j}\right| \rightarrow 0$ so that $a_{k, j}, b_{k, j} \rightarrow c_{k, j}$, and we need to determine both $\left|a_{k, j}-b_{k, j}\right|$ and $c_{k, j}$. This sort of analysis has been 
carried out by Traizet in a number of slightly different settings (e.g. [18, [17). Unfortunately for the brevity of this argument, while we can follow his general outline, he does not seem to have treated this precise case; fortunately, as all of the relevant information about the surfaces $M_{n}$ is in the development (9) (recall that the $d h$ development offers no substantive restrictions), we can give a full yet more elementary treatment directly from the equations (9).

The crucial condition is that $\int_{\gamma} g d h=\int_{\gamma} \overline{\frac{1}{g} d h}$ for every cycle $\gamma \subset M$. On the annuli $A_{n}$, this implies that

$$
F_{\Omega_{g d h}}(\alpha)-F_{\Omega_{g d h}}(\beta)=\overline{F_{\Omega_{g^{-1} d h}}(\alpha)-F_{\Omega_{g^{-1} d h}}(\beta)}
$$

for $\alpha, \beta \in P_{n} \cup Q_{n}$ : here the point is that any cycle on $M_{n}$ is homologous to a linear combination of arcs on the boundary connecting the vertical points of the Gauss map.

Let us normalize the setting. We focus on four consecutive points $\alpha-c \varepsilon, \alpha, \beta$, $\beta+d \varepsilon$; naturally, each of the points depends on the parameter $n$, and as $n \rightarrow \infty$, the points $\alpha-c \varepsilon$ and $\alpha$ converge to a node as do $\beta$ and $\beta+d \varepsilon$. In the natural notation, we compute

$$
F_{\Omega_{g d h}}(\beta)-F_{\Omega_{g d h}}(\alpha)=c_{0}+\frac{e^{i \pi / 4}}{2}(d-c) \varepsilon \log \varepsilon+\text { higher order terms }
$$

and

$$
F_{\Omega_{g^{-1} d h}}(\beta)-F_{\Omega_{g^{-1} d h}}(\alpha)=K_{-}\left(\overline{c_{0}}-\frac{e^{-i \pi / 4}}{2}(d-c) \varepsilon \log \epsilon+\text { higher order terms }\right) .
$$

Thus, in order that $F_{\Omega_{g d h}}(\beta)-F_{\Omega_{g d h}}(\alpha)=\overline{F_{\Omega_{g^{-1} d h}}(\beta)-F_{\Omega_{g^{-1} d h}}(\alpha)}$, we must have (from the singular term) that $K_{-} \rightarrow 1$ and $|d-c| \rightarrow 0$. As this computation holds for the interval between any pair of points coalescing to a node, we find that $\left|a_{k, j}-b_{k, j}\right| /$ $\left|a_{k^{\prime}, j}-b_{k^{\prime}, j}\right| \rightarrow 1$ as $n \rightarrow \infty$ for any choice of $k, k^{\prime}, j$ and $j^{\prime}$.

Remark 10. This last statement reflects that the sizes of the curves (as curves in space) being pinched are (asymptotically) identical.

Before turning our attention to the "small" intervals between $a_{k, j}$ and $b_{k, j}$, we readjust our notation, setting $b_{k, j}=a_{k, j}+\varepsilon+\eta_{k, j}$ where $\eta_{k, j}=o(\varepsilon(n))=o(\varepsilon)$ as $n \rightarrow \infty$. Also, being mindful of convergence issues that will eventually arise, we explicitly consider approximations (restricting the index $k$ to run between 1 and $M<\infty$ instead of between 1 and $\infty$ in the infinite product)

$$
\begin{gathered}
F_{\Omega_{g d h}}^{M}(\zeta)=e^{i \pi / 4} \int^{\zeta} \prod_{j=0}^{\varkappa}\left[\frac{z-a_{0, j}}{z-b_{0, j}}\right]^{1 / 2} \prod_{k=1}^{M} \prod_{j=0}^{\varkappa}\left[\frac{\left(z-a_{k, j}\right)\left(z-a_{-k, j}\right)}{\left(z-b_{k, j}\right)\left(z-b_{-k, j}\right)}\right]^{1 / 2} d z, \\
F_{\Omega_{g^{-1} d h}}^{M}(\zeta)=e^{-i \pi / 4} \int^{\zeta} \prod\left[\frac{z-b_{0, j}}{z-a_{0, j}}\right]^{1 / 2} \prod_{k=1}^{M} \prod_{j=0}^{\varkappa}\left[\frac{\left(z-b_{k, j}\right)\left(z-b_{-k, j}\right)}{\left(z-a_{k, j}\right)\left(z-a_{-k, j}\right)}\right]^{1 / 2} d z
\end{gathered}
$$

to the infinite vertex Schwarz-Christoffel map in (9). As the convergence of the "partial product" map in (10) is uniform in $M$ with estimates independent of $n$ (because $K_{-} \rightarrow 1$ and $P_{n} \cup Q_{n}$ converges to the nodes, uniformly in $n$ ), we see that the maps in (10) provide uniformly accurate approximations of the maps in (9) on compacta, for $M$ and $n$ sufficiently large. 
We consider, momentarily for fixed $k$, the quantity $F_{\Omega_{g d h}}^{M}\left(b_{k, j}\right)-F_{\Omega_{g d h}}^{M}\left(a_{k, j}\right)=$ $F_{\Omega_{g d h}}^{M}\left(a_{k, j}+\varepsilon+\eta_{k, j}\right)-F_{\Omega_{g d h}}^{M}\left(a_{k, j}\right)$ (for $M \gg k$ ) as $n \rightarrow \infty$, or equivalently, as $\varepsilon \rightarrow 0$. In the integrand, we can introduce the substitution $z=a_{k, j}+t\left(b_{k, j}-a_{k, j}\right)$ so that the factor

$$
\frac{z-a_{k, j}}{z-b_{k, j}}
$$

becomes $\frac{-t}{1-t}$. Moreover, using from the previous passage the estimate that $\left|b_{k^{\prime}, j^{\prime}}-a_{k^{\prime}, j^{\prime}}\right|=\varepsilon+o(n)$, the other factors

$$
\frac{z-a_{k^{\prime}, j^{\prime}}}{z-b_{k^{\prime}, j^{\prime}}}
$$

become $1+\frac{\varepsilon}{c_{k, j}-c_{k^{\prime}, j^{\prime}}}+o(\varepsilon)$ for $n$ sufficiently large: here recall that $c_{k}$ represents the position of the node which is the limit of the points $a_{k, j}$ and $b_{k, j}$. As a consequence, we compute that

$$
\begin{aligned}
F_{\Omega_{g d h}}^{M}\left(b_{k, j}\right)-F_{\Omega_{g d h}}^{M}\left(a_{k, j}\right)= & e^{i \pi / 4} \int_{0}^{1}\left(\frac{t}{1-t}\right)^{1 / 2} d t \\
& \times\left(1+\frac{\varepsilon}{2} \sum_{\substack{\left(k^{\prime}, j^{\prime}\right) \neq(k, j) \\
|k| \leq M}} \frac{1}{c_{k, j}-c_{k^{\prime}, j^{\prime}}}+o(\varepsilon)\right),
\end{aligned}
$$

where the sum runs over the terms in the integrand of $F_{\Omega_{g d h}}^{M}$ whose indices are not equal to $(k, j)$, with $k \leq M$.

Now, the computation of $F_{\Omega_{g^{-1} d h}}^{M}\left(b_{k, j}\right)-F_{\Omega_{g^{-1} d h}}^{M}\left(b_{k, j}\right)$ is analogous, yielding

$$
\begin{aligned}
F_{\Omega_{g^{-1} d h}}^{M}\left(b_{k, j}\right)-F_{\Omega_{g^{-1} d h}}^{M}\left(a_{k, j}\right)= & e^{-i \pi / 4} \int_{0}^{1}\left(\frac{1-t}{t}\right)^{1 / 2} d t \\
& \times\left(1-\frac{\varepsilon}{2} \sum_{\substack{\left(k^{\prime}, j\right) \neq(k, j) \\
|k| \leq M}} \frac{1}{c_{k, j}-c_{k^{\prime}, j^{\prime}}}+o(\varepsilon)\right) .
\end{aligned}
$$

Since $\int_{0}^{1}\left(\frac{t-1}{t}\right)^{1 / 2}=\int_{0}^{1}\left(\frac{t}{1-t}\right)^{1 / 2} d t$, we see that the horizontal period condition $F_{\Omega_{g d h}}\left(b_{k, j}\right)-F_{\Omega_{g d h}}\left(a_{k, j}\right)=\overline{F_{\Omega_{g^{-1} d h}}\left(b_{k, j}\right)-F_{\Omega_{g^{-1} d h}}\left(a_{k, j}\right)}$ provides that

$$
\mathcal{F}_{k, j}^{M}=\sum_{\substack{k^{\prime}, j^{\prime} \neq(k, j) \\|k| \leq M}} \frac{1}{c_{k, j}-c_{k^{\prime}, j^{\prime}}}=0 .
$$

(Evidently, these "forces" $\mathcal{F}_{k, j}^{M}$ converge to

$$
\mathcal{F}_{k, j}=\sum_{M=0}^{\infty} \sum_{\substack{\left.k^{\prime}, j^{\prime}\right) \neq(k, j) \\|k|=M}} \frac{1}{c_{k, j}-c_{k^{\prime}, j^{\prime}}}=0,
$$

but we prefer to continue to work with the approximations for a few more paragraphs, in order to interpret $\mathcal{F}_{k, j}$ as a gradient.) 
We have three final goals. We first aim to show that there is a unique configuration $\left\{c_{k, j}\right\}$ which satisfies (11), that this configuration consists of equally spaced points (to order $o(1)$ in $M$ ), and finally that this symmetric configuration is a nondegenerate critical point.

To begin, we observe that the "force" equations (11) may be interpreted as the vanishing of the gradient for the function $\widehat{H}\left(\left\{c_{k, j}\right\}\right)=-\prod_{\substack{(k, j) \neq\left(k^{\prime}, j\right) \\|k|=M}}\left|c_{k, j}-c_{k^{\prime}, j^{\prime}}\right|^{-1}$.

As the $c_{k, j}$ are periodic in $n$ (at least up to the cut-off $M$ ), we may regard this function as arising from a function $H$ with domain being the simplex $D=\{0=$ $\left.c_{0,0}<c_{0,1}<\cdots<c_{0, \varkappa}<1\right\}$. Clearly the function $H$ is proper on this simplex, and so we may expect an interior critical point at an interior global minimum. We now compute the Hessian of $H$, as equivalently, the differential of the map $\mathcal{F}^{M}: D \rightarrow \mathbf{R}^{\varkappa+1}$ given by $\mathcal{F}^{M}=\left(\mathcal{F}_{0,0}^{M}, \ldots, \mathcal{F}_{0 \varkappa}^{M}\right)$. This Hessian has the form

$$
\begin{aligned}
& \left(\begin{array}{cccc}
-\sum_{\substack{k, j) \neq(0,0) \\
|k| \leq M}}\left(c_{0,0}-c_{k, j}\right)^{-2} & \sum_{|k| \leq M}\left(c_{0,0}-c_{k, 1}\right)^{-2} & \cdots & \sum_{|k| \leq M}\left(c_{0,0}-c_{k, \varkappa}\right)^{-2} \\
\sum_{|k| \leq M}\left(c_{0,1}-c_{k, 0}\right)^{-2} & -\sum_{\substack{k, j) \neq(0,1) \\
|k| \leq M}}\left(c_{0,1}-c_{k, j}\right)^{-2} & \cdots & \sum_{|k| \leq M}\left(c_{0,1}-c_{k, \varkappa}\right)^{-2} \\
& & & -\sum_{\substack{(k, j) \neq(0, \varkappa) \\
|k| \leq M}}\left(c_{0, \varkappa}-c_{k, j}\right)^{-2}
\end{array}\right) \\
& =-\left[\sum_{j=0}^{\varkappa} \sum_{\substack{k, j) \neq(0, j) \\
|k| \leq M}}\left(c_{0, j}-c_{k, j}\right)^{-2}\right] I+\left(\sum_{|k| \leq M}\left(c_{0, i}-c_{k, j}\right)^{-2}\right)_{i, j=0}^{\varkappa} .
\end{aligned}
$$

As each row of the second matrix in (13) sums to the negative of the diagonal entry of the first matrix, we easily see that this Hessian is negative semi-definite with kernel coming only from a vector $(\lambda, \ldots, \lambda)$ with identical entries. As this vector $(\lambda, \ldots, \lambda)$ represents only a constant translation of the nodes to some direction or other, it is not a permissible deformation in $D$, since we required $c_{0,0}=0$. Thus this Hessian is negative definite on the (projectivized) domain $D$ of configurations of nodes. Thus each critical point of $-H$ has index 0 , and so, Morse theory applied to the cell $D$ implies that there is a unique critical point.

Finally let $c^{*}=\left(0, \frac{1}{\varkappa+1}, \frac{2}{\varkappa+1}, \ldots, \frac{\varkappa}{\varkappa+1}\right) \in D$ denote the configuration of equally spaced points in $D$. We observe that $\mathcal{F}_{i}^{M}\left(c^{*}\right)=o\left(\frac{1}{M}\right)$ (as $M$ tends to $\infty$ ). Thus, since the unique zero (say $c_{M}$ ) of $\mathcal{F}^{M}$ is a minimum of $H$, it is then uniformly bounded away from $\partial D$, and so we see that $c_{M} \rightarrow c^{*}$ as $M \rightarrow \infty$.

We conclude that the equally spaced point set $c^{*}$ is the unique limit configuration of the vertices $\left\{a_{k, j}, b_{k, j}\right\}$. Moreover, from the analysis above of the Hessians, we observe that $c^{*}$ is a nondegenerate zero of the map $\mathcal{F}_{0, j}: D \rightarrow \mathbf{R}^{\varkappa+1}$ (where we have now passed to the limit force equations (12)). Thus, by the implicit function theorem, there is a unique extension of this configuration to the unnoded case (9), yielding a zigzag, whose corresponding minimal surface has Scherk ends making a small positive angle between them. But as the standard Scherk examples are also such a family, and the family produced by the implicit function theorem is unique, we conclude that $\bar{M}_{n}=\overline{\mathcal{S}}_{t_{n}}$ for $n$ large (and some choice of $t_{n}$ ). This completes the proof of Proposition 9 . 


\section{The proofs of Theorems 1 and 2}

We are now in a position to prove Theorem 2 By the openness result in Proposition 4 the components of $\mathcal{M}_{\varkappa}$ are curves. By the properness result in Proposition 77. for each component $C$ of $\mathcal{M}_{\varkappa}$, the map $\theta: C \rightarrow\left(0, \frac{\pi}{2}\right]$ is a diffeomorphism. By the uniqueness result for small angles in Proposition 9, the only component of $\mathcal{M}_{\varkappa}$ for which the restriction of $\theta$ is onto is the component of Scherk examples. Hence, $\mathcal{M}_{\varkappa}$ consists of the component of Scherk examples, which proves Theorem 2 .

Assume now that $M$ is a connected minimal surface with $A(M)<3 \pi$. In this case, any limit tangent cone $C$ of $M$ is a cone over an integral varifold $F$ of $\mathbb{S}^{2}$, consisting of a finite number of geodesic segments joined at the finite number of vertices of $F$, and at each vertex $x_{0} \in F$, in a small neighborhood of $x_{0}$, the varifold $F$ consists of two geodesics crossing transversely. This fact follows immediately from our area growth assumption and the fact that when considered to be a current, the geodesic varifold $F$ has no boundary. From this local property at the vertices, we see immediately that $F$ is a finite union of circles and our area assumption implies that there are at most two such circles, counted with possible multiplicity. In particular, the area growth constant $A(M)=k \pi$, where $k=1$ or 2 .

If $A(M)=\pi$, then $M$ is a plane by the monotonicity formula for area. So, assume now that $A(M)=2 \pi$. In this case, any limit tangent cone at infinity for $M$ consists of two planes or a single plane of multiplicity two. Now we assume that $M$ has infinite symmetry group and we will prove that $M$ is a catenoid or one of the Scherk examples.

Since $M$ has infinite symmetry group, then $M$ is invariant under a continuous group of rotations or it is invariant under a screw motion symmetry. Assume that $M$ is not a catenoid, which is the only minimal surface of revolution. Since $M$ is invariant under a screw motion symmetry, one sees that it has a unique limit tangent cone $C(M)$ at infinity. It follows that $C(M)$ consists of two distinct planes or a single plane of multiplicity two. Since the screw motion symmetry of $M$ leaves $C(M)$ invariant, the screw motion composed with itself four times is a pure translation $\gamma: \mathbb{E}^{3} \rightarrow \mathbb{E}^{3}$, which, after a rigid motion and homothety, can be taken to be translation by the vector $(0,0,1)$ that lies in "both" planes in $C(M)$.

Consider the translational subgroup $\mathbb{Z}=\{(0,0, n) \mid n \in \mathbb{N}\}$ of $\mathbb{E}^{3}$, which leaves $M$ invariant. Note that if $\bar{M}=M / \mathbb{Z} \subset \mathbb{E}^{3} / \mathbb{Z}$ has finite topology, then Theorem 2 implies $M$ is a Scherk surface. So, it remains to show that $\bar{M}$ has finite topology. If $C(M)$ consists of two distinct planes $P_{1}, P_{2}$, then the facts that each plane has multiplicity 1 and $M$ is periodic imply that outside of some solid cylinder $\Delta$ around $P_{1} \cap P_{2}$, the surface $M$ consists of four graphs of small gradient over $\left(P_{1} \cup P_{2}\right)-\Delta$, which implies $\bar{M}$ has finite topology.

After a possible rotation around the $x_{3}$-axis, assume now that $C(M)$ is the $\left(x_{1}, x_{3}\right)$-plane $P$ (with multiplicity two as a locally finite integral varifold), and we will obtain a contradiction. Since $C(M)$ is unique and $M$ is invariant under $\mathbb{Z}$, there exists a $\mathbb{Z}$-invariant regular neighborhood $N(P)$ whose width around $P$ is a positive function $W\left(\left|x_{1}\right|\right)$ which grows sublinearly in the variable $\left|x_{1}\right|$, such that $M$ is contained in the interior of $N(P)$. Let $N\left(A_{x_{1}}\right)$ in $\mathbb{E}^{3} / \mathbb{Z}$ be the quotient regular neighborhood of $A_{x_{1}}$. Note that every annulus in $\mathbb{E}^{3} / \mathbb{Z}$ which is parallel to $A_{x_{1}}$ must intersect $\bar{M}$; otherwise $M$ is contained in a half-space in $\mathbb{E}^{3}$, which contradicts the half-space theorem [1], since $M$ is not a plane. For some $R>0$ large, the circle $\alpha_{R}=(0, R) \times \mathbb{R} / \mathbb{Z}$ lies outside of $N\left(A_{x_{1}}\right)$. For $\theta \in\left[0, \frac{\pi}{2}\right]$, let $A(\theta)$ be the (complete) 
vertical flat half-open annulus in $[0, \infty) \times[0, \infty) \times \mathbb{R} / \mathbb{Z}$ with boundary $\alpha_{R}$ which is parallel to the vector $(\cos (\theta), \sin (\theta), 0)$.

Without loss of generality, we may assume that $A(0) \cap \bar{M} \neq \emptyset$, and so, by the maximum principle for minimal surfaces, $A(0)$ intersects $\bar{M}$ transversely at some point. It follows that $A\left(\theta_{0}\right)$ also intersects $\bar{M}$ transversely at some point for some positive $\theta_{0}$ close to 0 . Since $A\left(\theta_{0}\right)$ intersects $N\left(A_{x_{1}}\right)$ in a compact set, then there is a nonempty compact subdomain $\Delta$ of $\bar{M}$ which lies in one of the bounded components of $N\left(A_{x_{1}}\right)-A\left(\theta_{0}\right)$. Hence, there exists a largest $\theta_{1}$ such that $A\left(\theta_{1}\right) \cap \bar{M} \neq \emptyset$ and such that at every point of this intersection, $\bar{M}$ locally lies on one side of $A\left(\theta_{1}\right)$. This contradicts the maximum principle for minimal surfaces, which completes the proof of Theorem 1

\section{REFERENCES}

[1] D. Hoffman and W. H. Meeks III. The strong halfspace theorem for minimal surfaces. Invent. Math., 101:373-377, 1990. MR1062966 (92e:53010)

[2] H. Karcher. Embedded minimal surfaces derived from Scherk's examples. Manuscripta Math., 62:83-114, 1988. MR0958255 (89i:53009)

[3] H. Karcher. Construction of minimal surfaces. Surveys in Geometry, pages 1-96, 1989. University of Tokyo, 1989, and Lecture Notes No. 12, SFB256, Bonn, 1989.

[4] H. Lazard-Holly and W. H. Meeks III. The classification of embedded doubly-periodic minimal surfaces of genus zero. Invent. Math., 143:1-27, 2001. MR.1802791 (2001m:53013)

[5] Hai-Ping Luo. Desingularizing the intersection between a catenoid and a plane. Ph.D. thesis, University of Massachusetts, Amherst, 1997.

[6] W. H. Meeks III. Geometric results in classical minimal surface theory, volume 8 of Surveys in Differential Geometry. International Press, edited by S.T. Yau, 2003. MR2039993 (2005b:53014)

[7] W. H. Meeks III. Global problems in classical minimal surface theory. In Global theory of minimal surfaces, pages 453-470. American Mathematical Society, Providence, RI, for the Clay Mathematics Institute, Cambridge, MA, edited by D. Hoffman, 2005. MR2167272 (2006f:53011)

[8] W. H. Meeks III and J. Pérez. Conformal properties in classical minimal surface theory. In Surveys of Differential Geometry IX - Eigenvalues of Laplacian and other geometric operators, pages 275-336. International Press, edited by Alexander Grigor'yan and Shing Tung Yau, 2004. MR 2195411

[9] W. H. Meeks III, J. Pérez, and A. Ros. Uniqueness of the Riemann minimal examples. Invent. Math., 131:107-132, 1998. MR1626477 (99b:53013)

[10] W. H. Meeks III and H. Rosenberg. The global theory of doubly periodic minimal surfaces. Invent. Math., 97:351-379, 1989. MR1001845 (90m:53017)

[11] W. H. Meeks III and H. Rosenberg. The maximum principle at infinity for minimal surfaces in flat three-manifolds. Comment. Math. Helvetici, 65:255-270, 1990. MR.1057243 (91d:53011)

[12] W. H. Meeks III and H. Rosenberg. The geometry of periodic minimal surfaces. Comment. Math. Helvetici, 68:538-578, 1993. MR.1241472 (95a:53011)

[13] J. Pérez, M. Rodríguez, and M. Traizet. The classification of doubly periodic minimal tori with parallel ends. Journal of Diff. Geometry, 69(3):523-577, 2005. MR2170278

[14] J. Pérez and M. Traizet. The classification of singly periodic minimal surfaces with genus zero and Scherk type ends. Transactions of the A.M.S. To appear.

[15] H. F. Scherk. Bemerkungen über die kleinste Fläche innerhalb gegebener Grenzen. J. $R$. Angew. Math., 13:185-208, 1835.

[16] R. Schoen. Uniqueness, symmetry, and embeddedness of minimal surfaces. J. of Differential Geometry, 18:791-809, 1983. MR0730928 (85f:53011)

[17] M. Traizet. Weierstrass representation of some simply-periodic minimal surfaces. Ann. Global Anal. Geom., 20:77-101, 2001. MR1846898(2002h:53015)

[18] M. Traizet. An embedded minimal surface with no symmetries. J. Differential Geometry, 60(1):103-153, 2002. MR1924593 (2004c:53008) 
[19] M. Weber and M. Wolf. Minimal surfaces of least total curvature and moduli spaces of plane polygonal arcs. Geom. Funct. Anal., 8:1129-1170, 1998. MR1664793 (99m:53020)

[20] M. Weber and M. Wolf. Teichmüller theory and handle addition for minimal surfaces. Annals of Math., 156:713-795, 2002. MR1954234 (2005j:53012)

[21] M. Wolf. Flat structures, Teichmüller theory and handle addition for minimal surfaces. In Global theory of minimal surfaces, pages 211-241. American Mathematical Society, Providence, RI, for the Clay Mathematics Institute, Cambridge, MA, edited by D. Hoffman, 2005. MR 2167261

Department of Mathematics, University of Massachusetts, Amherst, Massachusetts 01003

Department of Mathematics, Rice University, Houston, Texas 77005 